\title{
Topics in the Mathematical Physics of Cold Bose Gases
}

Jakob Yngvason

\begin{abstract}
In these notes of six lectures on selected topics in the theory of cold, dilute Bose gases, presented at the 5th Warsaw School of Statistical Physics in June 2013, the following topics are discussed: 1) The concept of BEC, 2) the ground state energy of a dilute Bose gas with short range interactions, 3) Gross-Pitaevskii theory and BEC in trapped gases, 4) Bose gases in rotating traps and quantized vortices, and 5) strongly correlated phases in the lowest Landau level generated by rapid rotation.
\end{abstract}

\section{Introduction}

Ingenious experimental techniques for cooling and trapping atoms [35] have since the mid 1990's opened a venue for studying the fascinating macroscopic quantum phenomena exhibited by of such systems [53, 55, 70]. These include Bose-Einstein condensation (BEC), superfluity, quantization of vorticity, and strong correlations produced by rapid rotations in Bose gases. Research on cold quantum gases is presently one of the most active areas of condensed matter physics.

On the theoretical side the subject is, in fact, quite old, going back to A. Einstein's paper on BEC in ideal (i.e., noninteracting) gases from 1924 [17. The theory of Bose gases with interactions began with N.N. Bogoliubov's fundamental work of 1947 5. This was followed by a period of considerable activity in this field in the late 1950's and early 60's. See, in particular 29, 16, 40. Due to the complexity of the quantum mechanical many-body problem, however, mathematically rigorous results were few and are still hard to get.

The challenge for mathematical physics is to start from a realistic many body Hamiltonian and derive the properties of its low energy states by rigor-

Jakob Yngvason

Faculty of Physics, University of Vienna, e-mail: jakob.yngvason@univie.ac.at 
ous mathematical analysis. Here substantial progress has been made in the past 15 years and a selection of such results is presented in these notes.

\section{The Concept of Bose-Einstein Condensation}

The basic facts about Bose-Einstein condensation can be summarized as follows:

- Under normal conditions the atoms of a gas are distributed among very many quantum states so that every single state is only occupied by relatively few atoms on the average.

- In BEC a single quantum state is occupied by a macroscopic number of atoms. This is possible if the atoms are bosons.

- For BEC in gases that are sufficiently dilute so that interactions can be ignored in first instance, extremely low temperatures, of the order $10^{-8}$ $\mathrm{K}$, are required.

Before discussing the general concept of BEC is is appropriate to review briefly the standard textbook treatment (see, e.g., [28]) for ideal gases.

\subsection{BEC in Ideal Bose Gases}

We consider a basis of single particle states, labelled by $k=0,1, \ldots 1$ and with energies $\varepsilon_{0} \leq \varepsilon_{1} \leq \varepsilon_{2} \cdots$. Classically, the $N$-particle states are specified by $N$-tuples, $\left(k_{1}, \ldots, k_{N}\right)$, but quantum mechanically, only the occupation numbers $\left(N_{0}, N_{1}, \ldots\right)$ with $\sum_{k} N_{k}=N$ matter. For fermions, only the values 0 or 1 are allowed, so macroscopic occupation of one state is excluded from the outset. For bosons, on the other hand, all values $N_{k}=0,1,2,3, \ldots$ are in principle possible. In the grand canonical ensemble the average occupation numbers are given by

$$
N_{k}= \begin{cases}z e^{-\varepsilon_{k} / k_{\mathrm{B}} T} & \text { for classical Boltzmann statistics } \\ \left(z^{-1} e^{\varepsilon_{k} / k_{\mathrm{B}} T}-1\right)^{-1} & \text { for Bose statistics }\end{cases}
$$

where the fugacity $z=e^{\mu / k_{\mathrm{B}} T} \geq 0$ (with $\mu$ the chemical potential) is determined by

$$
\sum_{k} N_{k}=N
$$

If $\varepsilon_{0}=0$ then $z<1$ for bosons. We now consider two specific examples.

${ }^{1}$ Classically the states are points $(p, q)$ in phase space that we think of as discretized for simpler comparison with the quantum case. 


\subsubsection{Particles in a box}

Suppose the particles are confined in a (large) rectangular box $\Lambda$ in $\mathbb{R}^{d}$ with volume $|\Lambda|$. In the thermodynamic limit where $N,|\Lambda| \rightarrow \infty$ with $\rho=N /|\Lambda|$ fixed, the energy values $\varepsilon_{k}$ scale like $|\Lambda|^{-2 / d}$ and the replacement

$$
\sum_{k} \rightarrow \int d \varepsilon D(\varepsilon)
$$

with the density of states $D(\varepsilon) \sim|\Lambda|$ appears reasonable. If classical statistics applies all $N_{k}$ are proportional to $z$, and hence

$$
\frac{N_{0}}{N}=\frac{1}{\int_{0}^{\infty} e^{-\varepsilon / k_{\mathrm{B}} T} D(\varepsilon) d \varepsilon} \sim \frac{1}{|\Lambda|} \rightarrow 0 \quad \text { for } \quad|\Lambda| \rightarrow \infty,
$$

i.e., there is no macroscopic occupation of the single particle ground state.

For a Bose gas the situation is different. Here the replacement (3) can in general only be used for the excited states and we write (again assuming $\left.\varepsilon_{0}=0\right)$

$$
\begin{aligned}
N_{0} & =\frac{1}{z^{-1}-1} \\
N & =N_{0}+\int_{\varepsilon_{1}}^{\infty} \frac{D(\varepsilon)}{z^{-1} e^{\varepsilon / k_{\mathrm{B}} T}-1} d \varepsilon
\end{aligned}
$$

keeping in mind that $\varepsilon_{1} \rightarrow 0$ as $|\Lambda| \rightarrow \infty$. We note also that the integral in Eq. (6) is monotonously increasing as $\varepsilon_{1} \rightarrow 0$ and also if $z \rightarrow 1$ from below. For a given $N$ and $\Lambda$, Eqs. (5) and (6) define $z=z(\rho ; N)$ and hence $N_{0}=N_{0}(\rho ; N)$. There are now two cases to consider:

1. BEC:

$$
\frac{1}{|\Lambda|} \int_{0}^{\infty} \frac{D(\varepsilon)}{e^{\varepsilon / k_{\mathrm{B}} T}-1} d \varepsilon=: \rho_{c}(T)<\infty .
$$

Then, if $\rho=N /|\Lambda|>\rho_{c}(T), z=z(\rho ; N) \rightarrow 1$ in the thermodynamic limit and

$$
\rho_{0}:=\frac{N_{0}}{|\Lambda|} \rightarrow \rho-\rho_{c}(T)>0 \quad \text { for } \quad N \rightarrow \infty .
$$

This is the case of BEC with a nonzero density $\rho_{0}$ of particles in the ground state.

2. No BEC:

$$
\frac{1}{|\Lambda|} \int_{0}^{\infty} \frac{D(\varepsilon)}{e^{\varepsilon / k_{\mathrm{B}} T}-1} d \varepsilon=\infty
$$

Then $z(\rho ; N)$ stays uniformly bounded away from 1 as $N \rightarrow \infty$, and hence $N_{0} / N \rightarrow 0$. Thus there is no BEC. 
From these considerations it is clear that it is the behavior of $D(\varepsilon)$ for $\varepsilon \rightarrow 0$ that matters for BEC in an ideal gas. In $d$ space dimensions $D(\varepsilon) \sim$ $|\Lambda| \varepsilon^{(d-2) / 2}$ and

$$
\frac{1}{|\Lambda|} \int_{\varepsilon_{1}}^{\infty} \frac{D(\varepsilon)}{z^{-1} e^{\varepsilon / k_{\mathrm{B}} T}-1} d \varepsilon \sim T^{d / 2} \sum_{\ell=1}^{\infty} \frac{z^{\ell}}{\ell^{d / 2}}
$$

Hence for $d=3$ there is BEC if

$$
\rho>\rho_{c}(T)=2,612\left(\frac{m k_{\mathrm{B}} T}{2 \pi \hbar^{2}}\right)^{3 / 2},
$$

a condition that can either be fulfilled at given $\rho$ by decreasing $T$, or at given $T$ by increasing $\rho$. The condition also be written as

$$
\rho^{-1 / 3}<\lambda_{\mathrm{dB}}=\left(2 \pi \hbar /\left(m k_{\mathrm{B}} T\right)\right)^{1 / 2}
$$

where the left-hand side is the mean particle distance and $\lambda_{\mathrm{dB}}$ is the thermal de Broglie wavelength. Since the ideal gas model can only be expected to be a reasonable approximation at low densities, low temperatures are required to achieve BEC.

In two and one dimensions, where $D(\varepsilon) /|\Lambda|=$ const. resp. $\sim \varepsilon^{-1 / 2}$, the integral (9) diverges at the lower boundary, or equivalently, the sum on the right-hand side of (10) diverges for $z \rightarrow 1$. Hence there is no BEC in the thermodynamic limit for $T>d^{2}$. It is interesting to note, however, that the divergence of the integral (9) is only logarithmic in $|\Lambda|$ since the lower integral boundary is $\varepsilon_{1} \sim|\Lambda|^{-2 / d}$. Hence, also for $d=2$ and $d=1$, there is BEC in the generalized sense that $N_{0} / N$ stays bounded away from zero in a modified thermodynamic limit where $|\Lambda| \sim N / \log N$ and hence $\rho \sim \log N$ for $N \rightarrow \infty$. This shows in particular that for a mathematically unambiguous definition of BEC it is important to specify how the parameters of the problem depend on $N$ as $N \rightarrow \infty$.

\subsubsection{Inhomogeneous gas in a trap}

Consider next a trap with a quadratic external potential in $\mathbb{R}^{d}$

$$
V(\mathbf{x}) \sim \omega^{2}|\mathbf{x}|^{2} .
$$

Here the density of states is

$$
D(\varepsilon) \sim \omega^{-d} \varepsilon^{(d-1)} .
$$

${ }^{2}$ In an ideal gas there is trivially complete BEC at $T=0$ with all particles sitting in the ground state. 
A natural 'thermodynamic limit' is defined by keeping $N \omega^{d}$, and hence $D(\varepsilon) / N$, fixed as $N \rightarrow \infty$.

Then

$$
\frac{1}{N} \int D(\varepsilon) \frac{1}{z^{-1} e^{\beta \varepsilon}-1} d \varepsilon \sim T^{d} \sum_{\ell=1}^{\infty} \frac{z^{\ell}}{\ell^{d}} .
$$

Hence there is BEC for $d=2$ and $d=3$ in this limit but not for for $d=1$. In three dimension the precise condition for BEC is

$$
N \omega^{3}>1,21\left(k_{\mathrm{B}} T / \hbar\right)^{3} .
$$

Although there is no BEC in a one dimensional trap for $N \omega$ fixed, there is generalized BEC in the sense mentioned above if $N \omega / \log N$ is kept fixed as $N \rightarrow \infty$ 34.

\subsection{The Concept of BEC for an Interacting Gas}

The many-body Hamiltonian for $N$ (spinless) Bosons with a pair interaction potential $v$ and an external potential $V$ has the form

$$
H_{N}=\sum_{i=1}^{N}\left\{-\frac{\hbar^{2}}{2 m} \nabla_{i}^{2}+V\left(\mathbf{x}_{i}\right)\right\}+\sum_{1 \leq i<j \leq N} v\left(\left|\mathbf{x}_{i}-\mathbf{x}_{j}\right|\right) .
$$

It acts on symmetric wave functions in $L^{2}\left(\mathbb{R}^{d N}\right)$. In the presence of interactions the energy eigenfunctions of $H_{N}$ are not simply symmetrized products of eigenfunctions of the one-particle operator as for a non-interacting system. Nevertheless, the concept of the average occupation, in a many-body state, of some given single particle state makes perfect sense, and can be used for a general definition of the concept of BEC. This is most conveniently expressed through the creation and annihilation operators $\hat{a}^{\dagger}(\varphi)$ und $\hat{a}(\varphi)$ for a single particle space $\varphi$, that act in the standard way on the symmetric Fock space built over the 1-particle space $L^{2}\left(\mathbb{R}^{d}\right)$.

If $\rho$ is any density matrix on Fock space, the average occupancy of $\varphi$ in the state corresponding to $\rho$ is

$$
N_{\varphi}=\operatorname{trace}\left(\rho \hat{a}^{\dagger}(\varphi) \hat{a}(\varphi)\right)=:\left\langle\hat{a}^{\dagger}(\varphi) \hat{a}(\varphi)\right\rangle_{\rho} .
$$

BEC in the many particle state $\langle\cdot\rangle_{\rho}$ means that the average occupancy is $O(N)$ for some 1-particle state $\varphi$, more precisely, for some $c>0$

$$
N_{\varphi} / N \geq c>0
$$

for all large $N$. Here 


$$
N=\sum_{i}\left\langle\hat{a}^{\dagger}\left(\varphi_{i}\right) \hat{a}\left(\varphi_{i}\right)\right\rangle_{\rho}
$$

with $\left\{\varphi_{i}\right\}$ an orthonormal basis in the 1-particle space, is the average total particle number in the state $\langle\cdot\rangle_{\rho}$.

It is clear that the definition is only mathematically precise if the dependence of the many-body state on $N$ is specified, and the macroscopically occupied state $\varphi$ will also in general depend on $N$.

A more concrete description can be given in terms of a partial trace of the density matrix $\rho$, namely the reduced 1-particle density matrix

$$
\rho^{(1)}\left(\mathbf{x}, \mathbf{x}^{\prime}\right)=\left\langle\hat{a}(\mathbf{x})^{\dagger} a\left(\mathbf{x}^{\prime}\right)\right\rangle_{\rho}
$$

If $\rho=|\Psi\rangle\langle\Psi|$ is a pure state given by an $N$ particle wave function $\Psi\left(\mathbf{x}_{1}, \ldots \mathbf{x}_{N}\right)$, then

$$
\rho^{(1)}\left(\mathbf{x}, \mathbf{x}^{\prime}\right)=N \int \Psi\left(\mathbf{x}, \mathbf{x}_{2}, \ldots \mathbf{x}_{N}\right) \bar{\Psi}\left(\mathbf{x}^{\prime}, \mathbf{x}_{2}, \ldots \mathbf{x}_{N}\right) d \mathbf{x}_{2} \cdots d \mathbf{x}_{N} .
$$

More generally, $\rho^{(1)}\left(\mathbf{x}, \mathbf{x}^{\prime}\right)$ is a convex combination of such expressions.

The 1-particle density matrix is the integral kernel of a trace class operator of trace $N$. It has a spectral decomposition

$$
\rho^{(1)}\left(\mathbf{x}, \mathbf{x}^{\prime}\right)=\sum_{i} N_{i} \varphi_{i}(\mathbf{x}) \bar{\varphi}_{i}\left(\mathbf{x}^{\prime}\right)
$$

with eigenvalues $N_{0} \geq N_{1} \geq \ldots$ and orthonormal $\varphi_{i}$.

BEC means that

$$
N_{0}=O(N)
$$

while the other $N_{i}$ are (in general) of lower order ${ }^{3}$ The eigenfunction $\varphi_{0}(\mathbf{x})$ of the integral kernel $\rho^{(1)}\left(\mathbf{x}, \mathbf{x}^{\prime}\right)$ to the highest eigenvalue is often referred to as the wave function of the condensate. Then $N_{0}\left|\varphi_{0}(\mathbf{x})\right|^{2}$ is the spatial density and $N_{0}\left|\tilde{\varphi}_{0}(\mathbf{p})\right|^{2}$, with $\tilde{\varphi}_{0}$ the Fourier transform of $\varphi_{0}$, the momentum density of the condensate.

For homogeneous gases in a box $\Lambda$ the wave function of the condensate can be expected to be the constant function $|\Lambda|^{-1 / 2}$. Since

$$
N_{0}=\iint \bar{\varphi}_{0}(\mathbf{x}) \rho^{(1)}\left(\mathbf{x}, \mathbf{x}^{\prime}\right) \varphi_{0}\left(\mathbf{x}^{\prime}\right) d \mathbf{x} d \mathbf{x}^{\prime}
$$

BEC means in this case

$$
|\Lambda|^{-1} \int_{\Lambda} \int_{\Lambda} \rho^{(1)}\left(\mathbf{x}, \mathbf{x}^{\prime}\right) d \mathbf{x} d \mathbf{x}^{\prime}=O(N)
$$

\footnotetext{
${ }^{3}$ If many eigenvalues are macroscopic one speak of fragmented condensation.
} 
instead of $O(1)$ if there is no BEC. This is called Off Diagonal Long Range Order.

In contrast to ideal gases, where there is always complete BEC in the ground state, the situation is quite different as soon as interaction is added. Here already the question of BEC in the ground state is highly nontrivial and it is only this case that will be discussed in the sequel. An essential first step in this direction is the understanding of the ground state energy.

\section{The Ground State Energy}

\subsection{The Scattering Length}

We consider the case $d=3$ and assume a rotationally symmetric pair interaction potential $v$ of short range between particles of mass $m$. The zero energy scattering equation for the two particle scattering in the relative coordinates is

$$
-\frac{\hbar^{2}}{m} \nabla^{2} \psi+v \psi=0
$$

Writing $\psi(\mathbf{x})=u(r) / r$ with $r=|\mathbf{x}|$ this is equivalent to

$$
-\frac{\hbar^{2}}{m} u^{\prime \prime}(r)+v(r) u(r)=0 .
$$

For $r$ larger than the range of $v$ the solution with $u(0)=0$ has the form

$$
u(r)=(\text { const. })(r-a)
$$

with a constant $a$ that is called the scattering length of $v$.

Equivalently,

$$
a=\lim _{r \rightarrow \infty}\left[r-\frac{u(r)}{u^{\prime}(r)}\right]
$$

and this is finite if $v$ decreases at least as $r^{-(3+\varepsilon)}$ at infinity. For $\psi(\mathbf{x})=u(r) / r$ we have outside of the range of $v$

$$
\psi(\mathbf{x})=\text { (const.) }\left(1-\frac{a}{r}\right) .
$$

If $v \geq 0$, then $0 \leq a \leq$ range of $v$. For a hard sphere potential $a$ is equal to the radius of the sphere.

If $v$ is not positive then $a$ can be negative, and if $-\frac{\hbar^{2}}{m} \nabla^{2}+v$ has bound states, $a$ can be much larger than the range of $v$.

If $v \geq 0$ the scattering length can be derived from a variational principle (see [44, Appendix C): 


$$
\frac{4 \pi \hbar^{2}}{m} a=\inf _{\psi} \int\left\{\frac{\hbar^{2}}{m}|\nabla \psi|^{2}+|\psi|^{2} v\right\} d^{3} \mathbf{x} .
$$

where the infimum is over all differentiable $\psi$ that tend to 1 at infinity. The infimum is attained for the zero energy scattering solution. The variational principle implies in particular 66.

$$
a \leq \frac{m}{4 \pi \hbar^{2}} \int v(r) d^{3} \mathbf{x}
$$

and the right-hand side is the first Born approximation of $a$ for a weak potential $v$.

For positive $v$ the scattering length determines also completely the ground state energy $E_{0}(2, L)$ of a pair of Bosons in a large box $\Lambda$ of side length $L \gg a$ :

$$
E_{0}(2, \Lambda) \approx \frac{4 \pi \hbar^{2}}{m} \frac{a}{L^{3}}
$$

In the Born approximation the right-hand side is just $\int v / L^{3}$, independent of $m$.

\subsection{The Ground State Energy of a Dilute Gas}

Consider now for $v \geq 0$ the Hamiltonian of $N$ Bosons in a box $\Lambda$ of side length $L$ :

$$
H_{N}^{\Lambda}=-\frac{\hbar^{2}}{2 m} \sum_{i=1}^{N} \nabla_{i}^{2}+\sum_{1 \leq i<j \leq N} v\left(\left|\mathbf{x}_{i}-\mathbf{x}_{j}\right|\right)
$$

Its ground state energy is

$$
E_{0}(N, L)=\inf _{\|\Psi\|=1}\left\langle\Psi, H_{N}^{\Lambda} \Psi\right\rangle
$$

and the energy per particle in the thermodynamic limit, $N=\rho L^{3} \rightarrow \infty$ with $\rho$ fixed, is

$$
e_{0}(\rho):=\lim E_{0}(N, L) / N
$$

This quantity is independent of the boundary conditions, but for energy bounds in a finite box one usually imposes Dirichlet or periodic conditions for the upper bound and Neumann conditions for the lower bound.

For the theory of dilute gases the low density asymptotics of $e_{0}(\rho)$ is of fundamental importance. Low density means here that

$$
\rho a^{3} \ll 1
$$


i.e., the scattering length is much smaller than the mean particle distance $\rho^{-1 / 3}$. The basic formula is

$$
e_{0}(\rho)=\frac{2 \pi \hbar^{2}}{m} a \rho(1+o(1))
$$

where the correction $o(1)$ tends to zero when $\rho a^{3} \rightarrow 0$.

A heuristic argument for this formula goes as follows:

"For a dilute gas only two body scattering matters", so we simply multiply the two-body energy (34) by the number of pairs, $N(N-1) / 2$, obtaining

$$
E_{0}(N, L) \approx \frac{N(N-1)}{2} E_{0}(2, L) \approx \frac{2 \pi \hbar^{2}}{m} N^{2} \frac{a}{L^{3}}=N \frac{2 \pi \hbar^{2}}{m} a \rho .
$$

Although this argument gives the correct leading term it is very far from a rigorous proof because the ground state can be highly correlated and it is not legitimate to regard the pairs $N(N-1) / 2$ as independent. Indeed, for $d=2$ the analogous argument gives the wrong answer: The ground state energy per particle is here $\sim \rho\left|\log \left(\rho a^{2}\right)\right|^{-1}\left[61\right.$, 49, while $E_{0}(2, L) \sim L^{-2}\left|\log \left(a^{2} / L^{2}\right)\right|^{-1}$ which multiplied by $N(N-1) / 2$ would give $e_{0}(\rho) \sim \rho\left|\log \left(a^{2} / L^{2}\right)\right|^{-1} \rightarrow 0$ for $L \rightarrow \infty$.

The formula 39 for the energy of a dilute Bose gas has an interesting history and it took almost 70 years to establish the leading term rigorously. The earliest reference is a paper of W. Lenz of 1929 [38] for a gas of hard spheres, using essentially the heuristic argument above. Bogoliubov's work of 1947 [5] was a milestone, but it was a perturbative result with $a$ replaced by its first and second Born approximations. In the 1950's and 60's several derivations were presented [29, 40, some containing also higher order terms:

$$
\frac{e_{0}(\rho)}{\left(2 \pi \hbar^{2} / m\right) a \rho}=\left[1+\frac{128}{15 \sqrt{\pi}}\left(\rho a^{3}\right)^{1 / 2}+8\left(\frac{4 \pi}{3}-\sqrt{3}\right)\left(\rho a^{3}\right) \log \left(\rho a^{3}\right)+O\left(\rho a^{3}\right)\right]
$$

These derivations all relied on some special assumptions about the ground state that have never been proved, or on the selection of special terms from a perturbation series which likely diverges. The only mathematically rigorous result in this period was due to F. Dyson [16, who in 1957 proved for a gas of hard spheres the estimates

$$
\frac{1+2 y^{1 / 3}}{\left(1-y^{1 / 3}\right)^{2}} \geq \frac{e_{0}(\rho)}{\left(2 \pi \hbar^{2} / m\right) a \rho} \geq \frac{1}{10 \sqrt{2}}
$$

with $y:=4 \pi a \rho^{3} / 3$. While the upper bound gives the desired result for $\rho a^{3} \rightarrow$ 0 , the lower bound (although obtained by an ingenious reasoning) is off the mark by a factor 14 .

Dyson's upper bound can be generalized to all short range interaction potentials $v \geq 0$ [45]. It is proved by a clever choice of a trial function, using the zero energy scattering solution for the two-body problem as an input. 
An asymptotically correct lower bound was not obtained until 1998 [48]:

Theorem 3.1 (Lower bound for g.s.e.)

$$
\frac{e_{0}(\rho)}{\left(2 \pi \hbar^{2} / m\right) a \rho} \geq\left(1-8.9 y^{1 / 17}\right) .
$$

The negative sign of the error term and the exponent $1 / 17$ result entirely from the technique of the proof and are not believed to reflect the true state of affairs. In the recent paper [37 the error term is improved to $-O\left(y^{1 / 3}|\log y|^{3}\right)$ by a modification of the method of 48 .

It is remarkable that the same formula, 390 , holds in two physically different regimes:

1. 'Hard potential', i.e., $v$ large within its range (in particular hard core). The energy is here mostly kinetic, due to the bending of the wave function down to small values when two points come close together. The ground state is highly correlated and simple perturbation theory is not applicable.

2. 'Soft potential', i.e., $v$ small everywhere. The energy is here mostly potential. Lowest order perturbation theory (with the uncorrelated, unperturbed state $\Psi_{0}=L^{-3 N / 2}$ ) gives

$$
e_{0}(\rho) \approx \frac{1}{2} \rho \int v(\mathbf{x}) d^{3} \mathbf{x}
$$

This is independent of $\hbar$ and $m$ and hence cannot be the right answer, but it is in accord with the first Born approximation for $a$.

It is still not entirely clear if the terms in (39) beyond leading order distinguish these regimes. See [19, 69, 25, for recent rigorous work on the second term in 411) (the "Lee-Huang-Yang term").

In the analysis of the ground state energy three different length scales are involved:

- The scattering length $a$.

- The mean particle distance $\rho^{-1 / 3} \gg a$.

- The 'healing length' $\ell_{c}$, defined by $1 / \ell_{c}^{2} \sim e_{0}(\rho)$.

For a dilute gas

$$
\ell_{c}=(a \rho)^{-1 / 2}=\left(\rho a^{3}\right)^{-1 / 6} \rho^{-1 / 3} \gg \rho^{-1 / 3} .
$$

Note that for fermions $\ell_{c} \sim \rho^{-1 / 3}$, i.e., much shorter than for bosons, which at low density and temperatures loose their individuality in the sense that the gas cannot be thought of as a system composed of well localized individual wave packets.

A key ingredient in the proof [48] of the lower bound is a lemma of Dyson [16], that allows the replacement of a short range 'hard' potential $v$ by a 
'soft' potential $a U_{R}$ of long range $R$, at the cost of sacrificing kinetic energy and interactions beyond nearest neighbours. Dyson's Lemma, in a way, thus transforms Regime 1 into Regime 2. Borrowing a small bit of the kinetic energy, perturbation theory applies, but one has to control the errors! For this it is necessary to divide $\Lambda$ into smaller boxed of side length $\ell$ that stays fixed as $L \rightarrow \infty$. In order for this strategy to work the parameters must satisfy

$$
a \ll R \ll \rho^{-1 / 3} \ll \ell \ll \ell_{c} .
$$

Optimizing the choice of $R, \ell$ and $\varepsilon$ leads to the error factor in (43) as shown in [48].

\section{Gross-Pitaevskii Theory}

Consider again the $N$-body Hamiltonian 17 with an external confining potential $V$. In this Section we choose units so that $\hbar=1, m=\frac{1}{2}$ and write

$$
H_{N}=\sum_{i=1}^{N}\left\{-\nabla_{i}^{2}+V\left(\mathbf{x}_{i}\right)\right\}+\sum_{1 \leq i<j \leq N} v\left(\left|\mathbf{x}_{i}-\mathbf{x}_{j}\right|\right) .
$$

The trap potential $V$ is assumed to be nonnegative, locally bounded, and tend to $\infty$ for $|\mathbf{x}| \rightarrow \infty$. In typical experiments with trapped gases $V$ can often be assumed to be quadratic, but this is not necessary and more general potentials will in particular become important in Sections 5 and 6. The interaction $v$ is, for the purpose of the mathematical model, supposed to be of finite range, rotationally symmetric, and nonnegative.

The external potential comes with a natural length scale $L_{\text {trap }}=e_{V}^{-1 / 2}$ where $e_{V}$ is the spectral gap between the ground state and the first excited state of $-\nabla^{2}+V$.

We are interested in the ground state properties of $H_{N}$, and in particular BEC, in the Gross-Pitaevskii (GP) limit where $N \rightarrow \infty$ with a fixed value of the GP interaction parameter

$$
g:=4 \pi N a / L_{\text {trap }}=e_{0}(\bar{\rho}) / e_{V}
$$

with $\bar{\rho}=N / L_{\text {trap }}^{3}$ a measure of the mean density. Note that since $\bar{\rho} a^{3} \sim$ $g / N^{2}=O\left(1 / N^{2}\right)$ if $g$ is fixed, the GP limit is a special case of a dilute limit.

The GP limit can be achieved in two ways:

- Keeping $a$ fixed and scaling the external potential $V$ so that $L_{\text {trap }} \sim N$ (not $\sim N^{1 / 3}$ as in the thermodynamic limit!), i.e, writing

$$
V(x)=N^{-2} V_{1}\left(N^{-1} x\right)
$$


with $V_{1}$ fixed.

- Keeping $V$ fixed and taking $a \sim N^{-1}$. The latter can be achieved by scaling the interaction potential: If $v_{1}$ is fixed with scattering length $a_{1}$, then

$$
v(r)=N^{2} v_{1}(N r)
$$

has scattering length $a=a_{1} N^{-1}$.

The alternatives (49) and (50) are completely equivalent although physically it may appear more natural to regard $v$ as fixed and scale $V$. Mathematically it is usually more convenient, however, to do the opposite. The ground state energy

$$
E_{0}(N, a)=\inf _{\|\Psi\|=1}\left\langle\Psi, H_{N} \Psi\right\rangle
$$

can then be regarded as a function of $N$ and $a=N^{-1} a_{1}$ for $V$ and $v_{1}$ fixed.

\subsection{The GP Energy Functional}

In the GP limit the essential features of the many-body ground state can be captured by minimizing a functional of functions on $\mathbb{R}^{3}$, the GP energy functional

$$
\mathcal{E}^{\mathrm{GP}}[\varphi]=\int_{\mathbb{R}^{3}}\left(|\nabla \varphi|^{2}+V|\varphi|^{2}+g|\varphi|^{4}\right),
$$

with the subsidiary condition $\int|\varphi|^{2}=1$.

The motivation for the term $g|\varphi|^{4}$ is the formula 39 for the energy of a dilute gas: With $\rho(\mathbf{x})=N|\varphi(\mathbf{x})|^{2}$ interpreted as a local density, we have

$$
N g \int|\varphi|^{4}=4 \pi a \int \rho^{2}
$$

and $4 \pi a \rho(\mathbf{x})^{2}$ is the interaction energy per unit volume. By standard methods it can be shown that this minimization problem has solution that is unique up to a phase factor that can be chosen so that the minimizer, denoted henceforth by $\varphi^{\mathrm{GP}}(\mathbf{x})$, is positive.

The minimizer is also the unique, nonnegative solution of the (time independent) Gross-Pitaevskii equation

$$
\left(-\nabla^{2}+V+2 g|\varphi|^{2}\right) \varphi=\mu^{\mathrm{GP}} \varphi
$$

with a Lagrange multiplier (chemical potential) $\mu^{\mathrm{GP}}$ to take care of the normalization $\int|\varphi|^{2}=1$.

The GP energy is

$$
E^{\mathrm{GP}}(g)=\mathcal{E}^{\mathrm{GP}}\left[\varphi^{\mathrm{GP}}\right]=\inf \left\{\mathcal{E}^{\mathrm{GP}}[\varphi]: \int|\varphi|^{2}=1\right\}
$$


and multiplying the GP equation by $\varphi^{\mathrm{GP}}$ and integrating we obtain

$$
\mu^{\mathrm{GP}}=E^{\mathrm{GP}}(g)+g \int\left|\varphi^{\mathrm{GP}}\right|^{4} .
$$

The GP energy functional can be obtained formally from the many body Hamiltonian by replacing $v\left(\mathbf{x}_{i}-\mathbf{x}_{j}\right)$ with $8 \pi a \delta\left(\mathbf{x}_{i}-\mathbf{x}_{j}\right)$ and making a Hartree type product ansatz for the many body wave function, i.e., writing

$$
\Psi\left(\mathbf{x}_{1}, \ldots, \mathbf{x}_{N}\right)=\varphi\left(\mathbf{x}_{1}\right) \cdots \varphi\left(\mathbf{x}_{N}\right) .
$$

This is not a proof, however, and the true ground state is not of this form (except for $v=0$ ). In particular, if $v$ has a hard core, then $\langle\Psi, H \Psi\rangle=\infty$ for all such product wave functions. Finite energy can in this case only be obtained for functions of the form

$$
\Psi\left(\mathbf{x}_{1}, \ldots, \mathbf{x}_{N}\right)=\varphi\left(\mathbf{x}_{1}\right) \cdots \varphi\left(\mathbf{x}_{N}\right) F\left(\mathbf{x}_{1}, \ldots, \mathbf{x}_{N}\right)
$$

with $F\left(\mathbf{x}_{1}, \ldots, \mathbf{x}_{N}\right)=0$ if $\left|\mathbf{x}_{i}-\mathbf{x}_{j}\right| \leq a$ for a pair $i \neq j$. The upper bound on the energy is, in fact, proved by using trial functions of this form with a judiciously chosen $F$ involving the zero-energy scattering solution of the two-body problem.

Remark: Formally, if $v_{1}(r) \sim \delta(\mathbf{x})$, then

$$
v(r)=N^{2} v_{1}(N r)=N^{-1+3 \beta} v_{1}\left(N^{\beta} r\right)
$$

independently of $\beta$. For a bona fide 3D potential $v_{1}$, on the other hand, the right-hand side depends on $\beta$. The GP limit corresponds to $\beta=1$. This is the case for which the scattering length is of the same order as the range of the potential. For $0<\beta<1$ and integrable $v$ one may expect that the interaction term is $\sim\left(\int v\right) \int|\varphi(\mathbf{x})|^{4} \mathrm{~d} \mathbf{x}$, i.e., the scattering length is replaced by its first Born approximation 4 The case $\beta=0$ is the Hartree limit, where the interaction term becomes $\iint|\varphi(\mathbf{x})|^{2} v(\mathbf{x}-\mathbf{y})|\varphi(\mathbf{y})|^{2} \mathrm{~d} \mathbf{x} \mathrm{d} \mathbf{y}$.

Let now $E_{0}(N, a)$ denote the many body ground state energy and $\rho^{(1)}\left(\mathbf{x}, \mathbf{x}^{\prime}\right)$ the one-particle density matrix of the ground state. Basic results in GP theory are the following rigorous theorems [45, 42, 64, 65]:

Theorem 4.1 (Energy asymptotics) If $N \rightarrow \infty$ with $g$ fixed (i.e., $a \sim$ $\left.N^{-1} L_{\text {trap }}\right)$, then

$$
\frac{E_{0}(N, a)}{N E^{\mathrm{GP}}(g)} \rightarrow 1
$$

Theorem 4.2 (BEC in GP limit) If $N \rightarrow \infty$ with $g$ fixed, then

$$
\frac{1}{N} \rho^{(1)}\left(\mathbf{x}, \mathbf{x}^{\prime}\right) \rightarrow \varphi^{\mathrm{GP}}(\mathbf{x}) \varphi^{\mathrm{GP}}\left(\mathbf{x}^{\prime}\right) \text { in trace norm. }
$$

\footnotetext{
${ }^{4}$ See the discussion in [18, 52 for the time dependent GP equation.
} 
In other words: There is complete BEC in the GP limit and the GP minimizer is the wave function of the condensate.

Corollary 4.1 In the GP limit the normalized particle density in the manybody ground state converges to $\left|\varphi^{\mathrm{GP}}(\mathbf{x})\right|^{2}$ and the normalized momentum density to $\left|\tilde{\varphi}^{\mathrm{GP}}(\mathbf{p})\right|^{2}$.

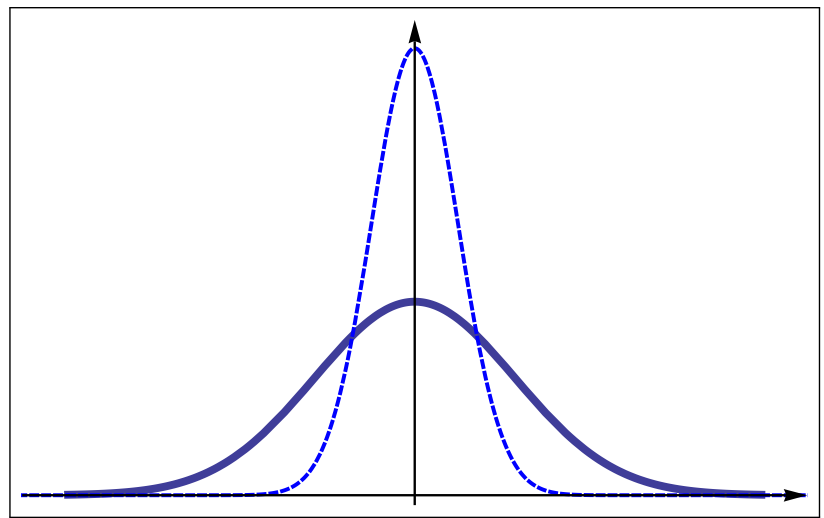

Fig. 1 The GP density (fat curve) and the density without $g|\varphi|^{4}$ (dotted).

\subsection{The 'Thomas-Fermi' approximation}

It is instructive and important to consider the properties of $\varphi^{\mathrm{GP}}$ as the interaction parameter $g$ varies, in particular the limiting case $g \gg 1$. Here it is convenient to assume that the trap potential $V$ is a homogeneous function of some order $s>0$, i.e., $V(\lambda \mathbf{x})=\lambda^{s} V(\mathbf{x})$ for $\lambda>0$.

For large $g$ salient features of the GP minimizer and energy can be estimated by the following 'back of the envelope' calculation: Let $R$ be the spatial extension of the condensate. Then $|\varphi|^{2} \sim R^{-3}$ and the three terms in the GP energy functional are of the following orders of magnitude:

$$
\int|\nabla \varphi|^{2} \sim R^{-2}, \quad \int V|\varphi|^{2} \sim R^{s}, \quad g \int|\varphi|^{4} \sim g R^{-3}
$$

For large $g$ the minimum of $R^{-2}+R^{s}+g R^{-3}$ is obtained for

$$
R \sim g^{1 /(s+3)}
$$

In particular for $s=2$ (quadratic trap) we obtain $R \sim g^{1 / 5}$. 
Note also that hat $\int|\nabla \varphi|^{2} \sim R^{-2} \sim g^{-2 /(s+3)}$ but the other terms of the energy are $\sim g^{s /(s+3)}$. Hence the kinetic term becomes irrelevant for $g \gg 1$.

To make this a little more precise we write $\mathbf{x}=g^{1 /(s+3)} \mathbf{x}^{\prime}$ and obtain

$$
\mathcal{E}^{\mathrm{GP}}[\varphi]=g^{s /(s+3)} \int_{\mathbb{R}^{3}}\left(g^{-(s+2) /(s+3)}\left|\nabla \varphi^{\prime}\right|^{2}+V\left|\varphi^{\prime}\right|^{2}+\left|\varphi^{\prime}\right|^{4}\right) \mathrm{d}^{3} \mathbf{x}^{\prime}
$$

with $\varphi^{\prime}\left(\mathbf{x}^{\prime}\right)=g^{3 / 2(s+3)} \varphi(\mathbf{x})$. Denoting $\left|\varphi^{\prime}\left(\mathbf{x}^{\prime}\right)\right|^{2}$ by $\rho\left(\mathbf{x}^{\prime}\right)$ we see that in the limit $g \rightarrow \infty$ the GP functional simplifies to the so-called Thomas-Fermi (TF) functiona $5^{5}$

$$
\mathcal{E}^{\mathrm{TF}}[\rho]=\int_{\mathbb{R}^{3}}\left(V \rho+\rho^{2}\right)
$$

with normalization $\int \rho=1$.

The minimizer can be displayed explicitly:

$$
\rho^{\mathrm{TF}}(\mathbf{x})=\frac{1}{2}\left[\mu^{\mathrm{TF}}-V(\mathbf{x})\right]_{+}
$$

where $\mu^{\mathrm{TF}}$ is chosen so that the normalization condition is fulfilled and $[t]_{+}=$ $t$ if $t \geq 0$ and zero otherwise. The corresponding TF energy is

$$
E^{\mathrm{TF}}=\inf _{\rho} \mathcal{E}^{\mathrm{TF}}[\rho]=\mathcal{E}^{\mathrm{TF}}\left[\rho^{\mathrm{TF}}\right]
$$

where the infimum is over all nonnegative $\rho$ with $\int \rho=1$. Moreover,

$$
\mu^{\mathrm{TF}}=E^{\mathrm{TF}}+2 \int\left(\rho^{\mathrm{TF}}\right)^{2} .
$$

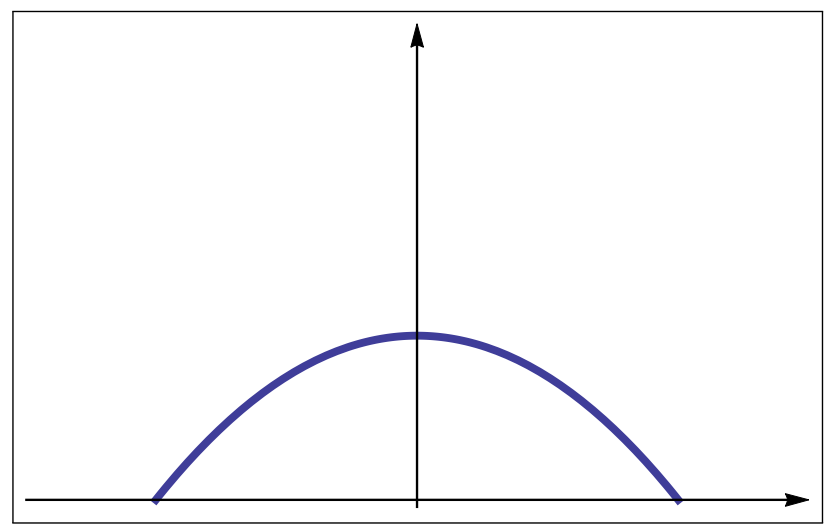

Fig. 2 The TF density.

5 This (somewhat unfortunate) denomination is due to a purely formal similarity with the density functional of Thomas-Fermi theory [41] for fermions. 
The TF minimizer and the TF ground state energy reproduce correctly the (suitably scaled) energy and particle density of the many body ground state of the many-body problem in the $N \rightarrow \infty, g \rightarrow \infty$ limit under the additional hypothesis that the gas remains dilute, i.e., $a^{3} \bar{\rho} \ll 1$ where $\bar{\rho}$ is the average density, of [44, Sec. 6.1. But it should be noted that BEC has not yet been proved in this limit, only in the GP limit where $g$ stays fixed, or grows at most very slowly with $N$.

\subsection{On the proof of BEC in the GP Limit}

The proof of BEC for trapped, dilute gases in [42] has two main ingredients:

- A refinement of the energy estimate (43). In fact, the deviation of (43) in [48] uses only part of the kinetic energy density, concentrated in a region in configuration space where two particles are close together. The matching upper bound to the energy implies that the neglected part of the kinetic energy is small and this implies an $L^{2}$-bound on the average kinetic energy in the complementary region.

- An extension of a classical Poincaré inequality that estimates an $L_{p}$ norm of the average value of a function by an $L_{q}$ norm of its gradient [46].

Theorem 4.2 holds for general trapping potentials $V$. The essential ideas can, however, be explained in a simplified setting, namely when the gas is confined in a box $\Lambda$ of side length $L$ with Neumann boundary conditions so the wave function of the condensate is the constant function $\varphi_{0}=L^{-3 / 2}$. We employ the notations $\mathbf{X}=\left(\mathbf{x}_{2}, \ldots, \mathbf{x}_{N}\right)$ and $\psi_{\mathbf{X}}(\mathbf{x})=\Psi_{0}(\mathbf{x}, \mathbf{X})$ with $\Psi_{0}$ the many-body ground state wave function. The depletion of the condensate is

$$
\begin{aligned}
& 1-N_{0} / N=1-\left(N L^{3}\right)^{-1} \int_{\Lambda} \int_{\Lambda} \rho^{(1)}\left(\mathbf{x}, \mathbf{x}^{\prime}\right) d \mathbf{x} d \mathbf{x}^{\prime} \\
&=\int_{\Lambda^{N-1}}\left\|\psi_{\mathbf{X}}(\cdot)-\left\langle\psi_{\mathbf{X}}\right\rangle\right\|_{L^{2}(\Lambda)}^{2} d \mathbf{X}
\end{aligned}
$$

where $\langle f\rangle=L^{-3} \int_{\Lambda} f$ denotes the average of a function $f$ over the box.

There is a simple Poincare inequality that estimates the deviation of a function from its mean value in terms of a norm of the gradient:

$$
\|f-\langle f\rangle\|_{L^{2}(\Lambda)}^{2} \leq C L^{2}\|\nabla f\|_{L^{2}(\Lambda)}^{2}
$$

This inequality is a straightforward consequence of the spectral decomposition of the Neumann Laplacian in the box. A more refined inequality allows to replaces the $L_{2}$ norm on the right-hand side with the $L_{6 / 5}$ norm, and combing this with Hölder's inequality one obtains for arbitrary (measurable) $\Omega \subset \Lambda$ 


$$
\|f-\langle f\rangle\|_{L^{2}(\Lambda)}^{2} \leq C_{1} L^{2}\|\nabla f\|_{L^{2}(\Omega)}^{2}+C_{2}\left|\Omega^{c}\right|^{2 / 3}\|\nabla f\|_{L^{2}(\Lambda)}^{2} .
$$

where $\Omega^{c}$ is the complement of $\Omega$ in $\Lambda$.

This inequality is now combined with a localization of the kinetic energy that is 'hidden' in the proof [48] of the asymptotic formula $e_{0} \sim \rho a(1+o(1))$ for the ground state energy:

While the total kinetic energy per particle is of the order $\rho a$,

$$
t_{\mathrm{kin}}(\Lambda)=\int_{\Lambda^{N-1}}\left\|\nabla \psi_{\mathbf{X}}\right\|_{L^{2}(\Lambda)}^{2} d \mathbf{X} \sim \rho a(1+o(1)),
$$

an inspection of the proof in [48] reveals that here is an $\Omega \subset \Lambda$ such that $\left|\Omega^{c}\right|=L^{3} \times o(1)$ and

$$
t_{\mathrm{kin}}(\Omega)=\int_{\Lambda^{N-1}}\left\|\nabla \psi_{\mathbf{X}}\right\|_{L^{2}(\Omega)}^{2} d \mathbf{X}=\rho a \times o(1) .
$$

Hence, if $N \rightarrow \infty$ with $g \sim N a / L$ fixed, then the combination of (69), (71), 72 and 73 gives

$$
1-N_{0} / N \leq L^{2} \rho a \times o(1)=\frac{N a}{L} \times o(1) \rightarrow 0 .
$$

\section{Rotating Bose Gases and Quantized Vortices}

\subsection{Quantization of Vorticity in a Superfluid}

Consider a fluid with velocity field $\mathbf{v}(\mathbf{r})$. The circulation around a closed loop $\mathcal{C}$ enclosing a domain $\mathcal{D}$ is, by Stokes,

$$
\oint_{\mathcal{C}} \mathbf{v} \cdot d \ell=\int_{\mathcal{D}}(\nabla \times \mathbf{v}) \cdot \mathbf{n} \mathrm{d} S .
$$

Hence nonzero circulation requires that the vorticity,

$$
\nabla \times \mathbf{v}
$$

is nonzero somewhere in $\mathcal{D}$. A region where $\nabla \times \mathbf{v} \neq 0$ is called a vortex.

The state of a superfluid can phenomenologically be described by a complex valued function ('order parameter') $\psi(\mathbf{x})=e^{\mathrm{i} \varphi(\mathbf{x})}|\psi(\mathbf{x})|$ satisfying a nonlinear Schrödinger Equation (the time dependent Gross-Pitaevskii equation)

$$
\mathrm{i} \hbar \partial \psi / \partial t=-\frac{\hbar^{2}}{2 m} \nabla^{2} \psi+F(\psi) \psi
$$


with $F(\psi)$ real valued ${ }^{6}$ The modulus squared, $\rho(\mathbf{x})=|\psi(\mathbf{x})|^{2}$, corresponds to the density of the superfluid while the phase determines the velocity of the flow:

$$
\mathbf{v}=\frac{\hbar}{m} \nabla \varphi
$$

To see this we note that just like for the linear Schrödinger equation, the nonlinear Schrödinger equation implies the continuity equation

$$
\partial \rho / \partial t+\nabla \cdot \mathbf{j}=0
$$

with the current density

$$
\mathbf{j}=\frac{\hbar}{2 m \mathrm{i}}(\bar{\psi} \nabla \psi-\psi \nabla \bar{\psi})
$$

Writing $\mathbf{j}(\mathbf{x})=\rho(\mathbf{x}) \mathbf{v}(\mathbf{x})$ one obtains $(78)$.

Since $\psi$ is single valued we have $\oint_{\mathcal{C}} \nabla \varphi \cdot d \ell=n 2 \pi$ with $n \in \mathbb{Z}$, so

$$
\oint_{\mathcal{C}} \mathbf{v} \cdot d \ell=n \frac{h}{m}
$$

This means that vorticity in a superfluid is quantized in units of $h / m$, as noted by L. Onsager in 1949 [50.

On the other hand, where the phase is nonsingular, i.e., where $|\psi(\mathbf{r})| \neq 0$, we have

$$
\nabla \times \mathbf{v}=0 .
$$

Generically, the complex function $\psi$ vanishes at most on one dimensional curves in $\mathbb{R}^{3}$ (points in $\mathbb{R}^{2}$ ) and by $(78)$ the flow is irrotational outside these vortex lines (resp. points). In contrast, rigid rotation with $\mathbf{v}(\mathbf{x})=\omega \times \mathbf{x}$ has $\nabla \times \mathbf{v}=2 \omega$ everywhere.

\subsection{The Many-body Hamiltonian in a Rotating Frame}

We now consider $N$ spinless bosons in trap potential, $V$, and with a pair interaction potential, $v$, like in Section 4 , but in addition we impose a uniform rotation with angular velocity $\boldsymbol{\Omega}_{\text {rot }}$ on the system, including the trap potential. The Hamiltonian in the rotating frame is

$$
H_{N}=\sum_{j=1}^{N}\left(-\frac{1}{2} \nabla_{j}^{2}+V\left(\mathbf{x}_{j}\right)-\mathbf{L}_{j} \cdot \boldsymbol{\Omega}_{\mathrm{rot}}\right)+\sum_{1 \leq i<j \leq N} v\left(\left|\mathbf{x}_{i}-\mathbf{x}_{j}\right|\right),
$$

${ }^{6}$ In a rotating frame $F$ is not real valued, cf. Eq. (89), but this leads only to the addition of the constant term $-2 \boldsymbol{\Omega}_{\text {rot }}$ to the vorticity. 
Here $\mathbf{L}_{j}=-\mathbf{i x}_{j} \times \nabla_{j}$ is the angular momentum of the $j$ th particle. The Hamiltonian can alternatively be written in the 'magnetic' form $7^{7}$

$$
H_{N}=\sum_{j=1}^{N}\left\{\frac{1}{2}\left(\mathrm{i} \nabla_{j}+\mathbf{A}\left(\mathbf{x}_{j}\right)\right)^{2}+V\left(\mathbf{x}_{j}\right)-\frac{1}{2} \Omega_{\mathrm{rot}}^{2} r_{j}^{2}\right\}+\sum_{1 \leq i<j \leq N} v\left(\left|\mathbf{x}_{i}-\mathbf{x}_{j}\right|\right)
$$

with the vector potential

$$
\mathbf{A}(\mathbf{x})=\Omega_{\mathrm{rot}} \times \mathbf{x}=\Omega_{\mathrm{rot}} r \mathbf{e}_{\theta}
$$

where $r=\left(x_{1}^{2}+x_{2}^{2}\right)^{1 / 2}$ denotes the distance from the rotation axis and $\mathbf{e}_{\theta}$ the unit vector in the angular direction. This way of writing the Hamiltonian corresponds to the splitting of the rotational effects into Coriolis and centrifugal forces. In the magnetic analogy the vector potential corresponds to a magnetic field $\mathbf{B}=\nabla \times \mathbf{A}=2 \boldsymbol{\Omega}_{\text {rot }}$.

A notable feature of the hamiltonian 83 is that, in contrast to the nonrotating case, the bosonic ground state is in general not the same as the absolute ground state (i.e., the ground state without symmetry requirement), and it need not be unique 63.

\subsection{Harmonic vs. Anharmonic Traps}

If $V$ is a harmonic oscillator potential in the direction $\perp$ to $\boldsymbol{\Omega}_{\mathrm{rot}}$, i.e.,

$$
V(\mathbf{x})=\frac{1}{2} \Omega_{\mathrm{trap}} r^{2}+V^{\|}\left(x_{3}\right)
$$

with $x_{3}$ the coordinate in the direction of the axis of rotation, then stability requires $\Omega_{\text {rot }}<\Omega_{\text {trap }}$. Rapid rotation means here that

$$
\Omega_{\text {rot }} \rightarrow \Omega_{\text {trap }}
$$

from below. On the other hand, if $V$ is anharmonic and increases faster than quadratically in the directions $\perp$ to $\boldsymbol{\Omega}_{\text {rot }}$, e.g. $V(\mathbf{x}) \sim\left(r^{s}+V^{\|}\left(x_{3}\right)\right)$ with $s>2$, then $\Omega_{\text {rot }}$ can in principle be as large as one pleases and 'rapid rotation' means simply that $\Omega_{\text {rot }} \rightarrow \infty$.

These two varieties of rapid rotation turn out to differ both physically and mathematically. The former, that we shall discuss in more detail in Section 6 , leads to an effective many-body Hamiltonian in the lowest Landau level of the magnetic kinetic energy term in (84) and bosonic analogues of the Fractional Quantum Hall Effect (see [20, 68, 8, 39]). On the other hand, in the case of rapid rotation in an anharmonic trap it is usually sufficient to

${ }^{7}$ Here and in the sequel we choose units so that the mass $m$ is 1 rather than $\frac{1}{2}$ as in Section 4. 
employ Gross-Pitaevskii (GP) theory for an effective description. We remark, however, that a small anharmonic term, appropriately tuned, also leads to interesting modifications of the Quantum Hall states of harmonic traps [57, 58. This will be discussed further in Sections 6.3 and 6.4.

\subsection{The Gross-Pitaevskii Limit Theorem with Rotation}

The following extensions of the GP limit theorems 4.1 and 4.2 to the rotating case was proved in 39:

Theorem 5.1 (GP limit and BEC at fixed $\boldsymbol{\Omega}_{\text {rot }}$ ) In the limit $N \rightarrow \infty$ with $g=4 \pi N a / L_{\text {trap }}$ and $\boldsymbol{\Omega}_{\mathrm{rot}}$ fixed the ground state energy of 83 converges to the minimum energy of the Gross-Pitaevskii energy functional with rotation:

$$
\begin{aligned}
\mathcal{E}^{\mathrm{GP}}[\psi] & =\int_{\mathbb{R}^{3}}\left\{\frac{1}{2}|\nabla \psi|^{2}+V|\psi|^{2}-\boldsymbol{\Omega}_{\mathrm{rot}} \cdot \bar{\psi} \mathbf{L} \psi+g|\psi|^{4}\right\} \mathrm{d}^{3} \mathbf{x} \\
& =\int_{\mathbb{R}^{3}}\left\{\frac{1}{2}|(\mathrm{i} \nabla+\mathbf{A}) \psi|^{2}+\left(V-\frac{1}{2} \Omega_{\mathrm{rot}}^{2} r\right)|\psi|^{2}+g|\psi|^{4}\right\} \mathrm{d}^{3} \mathbf{x}
\end{aligned}
$$

with the normalization condition $\int_{\mathbb{R}^{3}}|\psi|^{2}=1$.

Moreover, there is (possibly fragmented) BEC in this limit in the sense that every one-particle density matrix obtained as the limit of normalized oneparticle density matrices of ground states of (84), is a convex combination of projectors onto minimizers of the GP functional.

Every GP minimizer solves the GP equation

$$
\left\{-(\nabla-\mathrm{i} \mathbf{A})^{2}+\left(V-\frac{1}{4} \Omega_{\mathrm{rot}}^{2} r^{2}\right)+2 g|\psi|^{2}\right\} \psi=\mu^{\mathrm{GP}} \psi,
$$

but in contrast to the non-rotating case, the minimizer need not be unique up to a constant phase factor. The reason is a new feature compared to the non-rotating case, namely the possible occurrence of vortices that may break rotational symmetry, even if $V$ depends only on $r$ besides $x_{3}$.

The proof of the GP limit theorem in 43 . uses the technique of coherent states and is rather different from the proof in [45, 42, for the non-rotating case. The reason is that the splitting of space into boxes where the system is approximately homogeneous, as used in the previous proof, is not applicable in the presence of the global vector potential $\mathbf{A}$.

The GP equation 89 and its vortex solutions is a subject of its own that can be studied independently of the many-body problem. See the monograph [1] and the review article [20] where a large number of references can be found. The most detailed results are for the two-dimensional GP equation, i.e., when $\psi$ depends only on the coordinates $\left(x_{1}, x_{2}\right)$ in the plane perpendicular to the angular velocity, and in the asymptotic regime when $g \rightarrow \infty$. In 
particular, the two-dimensional GP equation with a quadratic trap potential $V(\mathbf{r}) \sim r^{2}$ has been studied in [2, 30, 31]. More general homogeneous trapping potentials are discussed, e.g., in [12, 56]. In this regime powerful techniques, in particular from Ginzburg-Landau theory [59] ('vortex ball constructions'), can be applied. The case of a fixed, finite value of $g$ is much less explored but several important general results were obtained in 62 .

It is convenient and customary to write the coupling strength $g=$ $4 \pi N a / L_{\text {trap }}$ as $1 / \varepsilon^{2}$ with

$$
\varepsilon=g^{-1 / 2}
$$

which is small if $N a / L_{\text {trap }}$ is large. This parameter can be thought of as the ratio of the healing length $\ell_{c} \sim(a \bar{\rho})^{-1 / 2}$ at mean density $\bar{\rho}=N / L_{\text {trap }}^{3}$ to the length scale $L_{\text {trap }}$ of the trap.

In anharmonic traps, where $\Omega_{\text {rot }}$ can be arbitrary, we shall in particular be interested in the asymptotic regime where both $g$ and $\Omega_{\text {rot }}$ are large.

\subsubsection{Status of GP for rapid rotation}

The rigorous derivation of the GP equation from the many-body problem has so far only been achieved for $\Omega_{\mathrm{rot}}$ and $\varepsilon$ fixed. For rapid rotation the GP description can break down both in harmonic and anharmonic traps, because the convergence of the many-body quantities to the GP quantities need not hold uniformly in the parameters. The exact limitations, that may depend on the quantities of interest, have not yet been established rigorously. For instance, even in the non-rotating case BEC has not been proved in the TF limit, i.e., when $g \rightarrow \infty$, although there is a limit theorem for the energy and density (cf. Section 4.2), provided the gas remains dilute in the limit. For rotating gases in anharmonic traps an analogous result was proved in [6]:

Theorem 5.2 (TF limit with rotation) If $N \rightarrow \infty$ with $\Omega_{\text {rot }} \rightarrow \infty$ and $\varepsilon \rightarrow 0$ but the gas remaining dilute (in the sense that mean density is $\ll a^{-3}$ ), then the TF approximation, i.e., the GP energy functional without the kinetic term $\frac{1}{2}|(\mathrm{i} \nabla+\mathbf{A}) \psi|^{2}$, gives the leading term in the ground state energy as a function of $\Omega_{\mathrm{rot}}$ and $\varepsilon$.

The leading TF term, however, does not exhibit vortices which are due to the kinetic term and have only an effect on the energy to next than leading order.

In harmonic traps the limit $\Omega_{\text {rot }} \rightarrow \Omega_{\text {trap }}$ has so far been studied in two steps: In the first step a limit has been considered in which an effective 2D many-body model in the lowest Landau level with contact interactions emerges [39. In the second step sufficient conditions for the validity of a GP limit for this effective model have been derived 47. This will be discussed in more detail in Section 6.2. A direct derivation of the GP energy functional 
in the lowest Landau level from the full 3D many-body Hamiltonian (83) has not yet been carried out.

\subsection{Two-Dimensional GP Vortices}

This subsection provides some heuristic background for understanding the occurrence of vortices in the case of the two-dimensional GP equation.

The first thing to note is that for sufficiently small rotational velocities the condensate stays at rest in the inertial frame and thus appears to rotate opposite to $\boldsymbol{\Omega}_{\mathrm{rot}}$ in the rotating frame. This is a manifestation of superfluidity: A normal fluid would pick up the rotational velocity of the container and in equilibrium the fluid would be at rest in the rotating frame.

In the rotating frame the operator of the velocity is $-\mathrm{i} \nabla-\mathbf{A}(\mathbf{r})$. The constant wave function, that (in a 'flat' trap) minimizes the GP energy functional (with zero energy in excess of the interaction energy) for small $\Omega_{\text {rot }}$, thus has, in the rotating frame, the velocity

$$
\mathbf{v}(\mathbf{r})=-\mathbf{A}(\mathbf{r})=-\boldsymbol{\Omega}_{\mathrm{rot}} \times \mathbf{r}=-\Omega_{\mathrm{rot}} r \mathbf{e}_{\theta},
$$

where $\mathbf{e}_{\theta}$ denotes the unit vector with respect to the angular variable. Note that the kinetic energy corresponding to this velocity is exactly compensated by the centrifugal term $-\frac{1}{2} \Omega_{\mathrm{rot}}^{2} r^{2}$ in the GP energy functional 88 .

At higher rotational velocities the condensate responds by creating vortices whose velocity field may partly compensate the term - A of the velocity and hence reduce the kinetic energy. This reduction does not come for free, however, because the creation of a vortex is accompanied by a redistribution of the density and hence an increase in interaction energy.

To estimate these competing effects let us consider the case of large $g$, i.e., small $\varepsilon=g^{-1 / 2}$ and a trap with effective radius $R$. A vortex of degree $d$ located at the origin can, for the purpose of this heuristic discussion, be approximated by the ansatz

$$
\psi(r, \theta)=f(r) \exp (\mathrm{i} \theta d)
$$

with

$$
f(r) \sim\left\{\begin{array}{l}
r^{d} \text { if } \quad 0 \leq r \lesssim r_{\mathrm{v}} \\
R^{-1} \text { if } \quad r_{\mathrm{v}} \lesssim r \leq R
\end{array}\right.
$$

where $r_{\mathrm{v}}$ is the radius of the vortex core where the density is small. Now the component of the velocity in the direction of $\mathbf{e}_{\theta}$ is

$$
\mathbf{v}(\mathbf{r})_{\theta}=\left(\frac{d}{r}-\Omega_{\mathrm{rot}} r\right) \mathbf{e}_{\theta} .
$$


The change in kinetic energy compared to the vortex free case, $d=0$, is therefore

$$
\sim R^{-2} \int_{r_{\mathrm{v}}}^{R}\left[(d / r)^{2}-d \Omega_{\mathrm{rot}}\right] r d r+O(1)=R^{-2} d^{2}\left|\log \left(r_{\mathrm{v}} / R\right)\right|-\frac{1}{2} d \Omega_{\mathrm{rot}}+O(1) .
$$

On the either hand the change in interaction energy through the creation of the vortex is

$$
\sim \frac{1}{\varepsilon^{2}}\left(r_{\mathrm{v}} / R\right)^{2}
$$

Optimizing the total energy change w.r.t. $r_{\mathrm{v}}$ gives a vortex radius of the order of the healing length, i.e.

$$
r_{\mathrm{v}} \sim \varepsilon R
$$

and an interaction energy increase $\sim R^{-2}$. A vortex of degree $d=1$ becomes energetically favorable when this is outweighed by a decrease in kinetic energy, i.e., if

$$
R^{-2}|\log \varepsilon|-\frac{1}{2} \Omega_{\mathrm{rot}}+O(1) R^{-2}<0
$$

which means

$$
\Omega_{\text {rot }} \gtrsim O(1) R^{-2}|\log \varepsilon| .
$$

We also see that $d$ vortices of degree 1 , ignoring their interaction, have energy $\sim d\left(R^{-2}|\log \varepsilon|-\Omega_{\text {rot }}\right)$ while a vortex of degree $d$ has energy $R^{-2} d^{2}|\log \varepsilon|-$ $d \Omega_{\text {rot }}$. Hence it is energetically favorable to 'split' a $d$-vortex into $d$ pieces of 1 -vortices, breaking the rotational symmetry.

These heuristic considerations are confirmed by a detailed analysis for 'slowly' rotating gases, i.e., $\Omega_{\text {rot }}=O(|\log \varepsilon|)[2,3$, , 30, 31, 56. In a 'flat' trap vortices start to appear for $\Omega_{\mathrm{rot}} R^{2}=\pi|\log \varepsilon|$ and for

$$
|\log \varepsilon|+(d-1) \log |\log \varepsilon|<\Omega_{\mathrm{rot}} R^{2} / \pi \leq|\log \varepsilon|+d \log |\log \varepsilon|
$$

there are exactly $d$ vortices of degree 1 . In a homogeneous trap, $V(r) \sim r^{s}$, the effective radius $R$ of the condensate can be estimated by equating the potential energy in the trap and the interaction energy, i.e., $R^{s} \sim \varepsilon^{-2} R^{2}\left(R^{-1}\right)^{4}$, which leads to

$$
R \sim \varepsilon^{-2 /(s+2)}
$$

and thus the critical velocity for the creation of a vortex is

$$
\sim \varepsilon^{4 /(s+2)}|\log \varepsilon| .
$$

In particular, for a harmonic trap with $s=2$ the critical rotational velocity is $\sim \varepsilon|\log \varepsilon|$. The decrease with $\varepsilon$ of the critical velocity for creating a vortex in such traps is entirely due to the fact that the interaction spreads out the mass and decreases the density. In a trap with hard walls so that the radius is fixed the critical velocity is $\sim|\log \varepsilon|$ and hence increases when $\varepsilon \rightarrow 0$. 


\subsection{GP Theory for Rapid Rotation, Anharmonic Traps}

In this subsection we consider the effects of rapid rotation on the GP minimizer in a $2 \mathrm{D}$ anharmonic trap. It should be noted that $2 \mathrm{D}$ results are of experimental relevance also in 3D: 1) to systems that are strongly confined in one direction so that the motion is effectively two-dimensional, or 2) for traps that are highly elongated in the direction of the rotational axis so that the properties of the condensate are approximately independent of the coordinate in this direction.

For mathematical simplicity we consider a 2D 'flat', disc-shaped trap with rigid boundary and radius 1 . Some comments on more general traps will be made at the end.

The GP energy functional on the unit $\operatorname{disc} \mathcal{D} \subset \mathbb{R}^{2}$ is

$$
\mathcal{E}^{\mathrm{GP}}[\psi]=\int_{\mathcal{D}}\left\{\frac{1}{2}|(\mathrm{i} \nabla+\mathbf{A}) \psi|^{2}-\frac{1}{2} \Omega_{\mathrm{rot}}^{2} r^{2}|\psi|^{2}+\frac{1}{\varepsilon^{2}}|\psi|^{4}\right\} \mathrm{d}^{2} \mathbf{r}
$$

where $\mathbf{A}(\mathbf{r})=\Omega_{\text {rot }} r \mathbf{e}_{\theta}$.

As mentioned in the previous subsection one can prove that if $\Omega_{\text {rot }} \leq$ $\Omega_{c_{1}}|\log \varepsilon|+O(\log |\log \varepsilon|)$ for a certain $\Omega_{c_{1}}$ there is a finite number of vortices, even as $\varepsilon \rightarrow 0$. For larger $\Omega_{\text {rot }}$ the number of vortices is unbounded as $\varepsilon \rightarrow 0$. If $\Omega_{\text {rot }}$ is still $O(|\log \varepsilon|)$ the vortices are not uniformly distributed, however. This transition region has recently been analyzed in [13].

For $\Omega_{\text {rot }} \gg|\log \varepsilon|$ new phenomena appear at two critical velocities, namely for $\Omega_{\text {rot }} \sim 1 / \varepsilon$ and $\Omega_{\text {rot }} \sim 1 /\left(\varepsilon^{2}|\log \varepsilon|\right)$ respectively:

If $\Omega_{\text {rot }}=O(1 / \varepsilon)$ the centrifugal term $-\left(\Omega_{\text {rot }}^{2} / 2\right) r^{2}|\psi(\mathbf{r})|^{2}$ and the interaction term $\left(1 / \varepsilon^{2}\right)|\psi(\mathbf{r})|^{4}$ are comparable in size and the centrifugal forces influence the bulk shape of the condensate. The kinetic energy term $\frac{1}{2}|(\mathrm{i} \nabla+\mathbf{A}(\mathbf{r})) \psi(\mathbf{r})|^{2}$ is formally also of order $1 / \varepsilon^{2}$ if $\Omega_{\text {rot }} \sim 1 / \varepsilon$, but it turns out that its contribution to the energy is, in fact, of lower order, namely $\sim \Omega_{\text {rot }}|\log \varepsilon|$, because a lattice of vortices emerges as $\varepsilon \rightarrow 0$. The velocity field generated by the vortices compensates partly the field $-\mathbf{A}(\mathbf{r})$ generated by the rotation.

For $\Omega_{\text {rot }} \geq \Omega_{c_{2}} 1 / \varepsilon$ the centrifugal forces deplete strongly the density in a 'hole' of radius

$$
R_{\mathrm{h}}=1-c\left(\Omega_{\mathrm{rot}} \varepsilon\right)^{-1}
$$

around the rotation axis and the bulk of the condensate is concentrated in a thin annulus of thickness $\sim\left(\varepsilon \Omega_{\text {rot }}\right)^{-1}$. As long as $\Omega_{\text {rot }} \ll 1 /\left(\varepsilon^{2}|\log \varepsilon|\right)$, however, the annulus still contains a lattice of vortices, but if $\Omega_{\text {rot }}>$ $\Omega_{c_{3}} 1 /\left(\varepsilon^{2}|\log \varepsilon|\right)$ the high density of the condensate in the annulus make vortices too costly. A transition to a 'giant vortex' state [32, 22, 33, 21, 23, takes place where all vorticity is concentrated in the 'hole' but the bulk of the condensate is vortex free. 
We now discuss these results in more detail, starting with the parameter region $|\log \varepsilon| \ll \Omega_{\text {rot }} \ll 1 /\left(\varepsilon^{2}|\log \varepsilon|\right)$ where the following holds [15]:

Theorem 5.3 (Energy to subleading order) Let $E^{\mathrm{GP}}$ denote the GP energy, i.e., the minimum of the GP energy functional. Let $E^{\mathrm{TF}}$ denote the minimal energy of the GP functional without the kinetic term.

If $|\log \varepsilon| \ll \Omega_{\text {rot }} \ll 1 / \varepsilon$, then

$$
E^{\mathrm{GP}}=E^{\mathrm{TF}}+\frac{1}{2} \Omega_{\mathrm{rot}}\left|\log \left(\varepsilon^{2} \Omega_{\mathrm{rot}}\right)\right|(1+o(1)) .
$$

If $1 / \varepsilon \lesssim \Omega_{\text {rot }} \ll 1 /\left(\varepsilon^{2}|\log \varepsilon|\right)$ then

$$
E^{\mathrm{GP}}=E^{\mathrm{TF}}+\frac{1}{2} \Omega_{\mathrm{rot}}|\log \varepsilon|(1+o(1)) .
$$

In both cases the energy corresponds to a uniform distribution of vorticity in a the form of a vortex lattice in the bulk of the condensate.

\subsubsection{An electrostatic analogy}

The upper bound to the energy in Theorem 5.3 is based on a variational ansatz that is motivated by an electrostatic analogy.

We write points $\mathbf{r}=(x, y) \in \mathbb{R}^{2}$ as complex numbers, $\zeta=x+\mathrm{i} y$, and consider a lattice of points $\zeta_{j}$. Placing a vortex of degree 1 at each point $\zeta_{j}$ leads to a trial function for the GP energy of the form

$$
\psi(\mathbf{r})=f(\mathbf{r}) \exp \{\mathrm{i} \varphi(\mathbf{r})\}
$$

where $f$ is real valued with a zero at each of the points $\zeta_{j}$ and the phase factor is

$$
\exp \{\mathrm{i} \varphi(\mathbf{r})\}=\prod_{j} \frac{\zeta-\zeta_{j}}{\left|\zeta-\zeta_{j}\right|}
$$

Now

$$
|(\mathrm{i} \nabla+\mathbf{A}) \psi|^{2}=|\nabla f|^{2}+f^{2}|\mathbf{A}-\nabla \varphi|^{2}
$$

and

$$
\varphi=\sum_{j} \arg \left(\zeta-\zeta_{j}\right)
$$

The phase $\arg z$ of a complex number is the imaginary part of the complex logarithm which is an analytic function on the complex plane (suitably cut). The Cauchy-Riemann equations for the real and imaginary part of an analytic functions imply

$$
|\mathbf{A}-\nabla \varphi|^{2}=\left|\Omega_{\text {rot }} r \mathbf{e}_{r}-\nabla \chi\right|^{2}
$$

where

$$
\chi(\mathbf{r})=\sum_{j} \log \left|\mathbf{r}-\mathbf{r}_{j}\right| .
$$


But

$$
\mathbf{E}(\mathbf{r}):=\Omega_{\mathrm{rot}} r \mathbf{e}_{r}-\nabla \chi(\mathbf{r})
$$

has a simple physical interpretation: It can be regarded as an 'electric field' generated by a uniform charge distribution of density $\Omega_{\text {rot }} / \pi$ together with unit 'charges' of opposite sign at the positions of the vortices, $\mathbf{r}_{j}$. The integral of $|\mathbf{E}(\mathbf{r})|^{2}$ is the corresponding electrostatic energy.

To construct a trial function for an upper bound to the GP energy we distribute the vortices over the unit disk so that the vorticity per unit area is $\Omega_{\text {rot }} / \pi$. (This is really $2 \Omega_{\text {rot }} \cdot m / h$.) Thus every vortex sits at the center $\mathbf{r}_{j}$ of lattice cell $Q_{j}$ of area $\left|Q_{j}\right|=\pi / \Omega_{\text {rot }}$, surrounded by a uniform charge distribution of the opposite sign so that the total charge in the cell is zero.

If the cells were disc-shaped, then Newton's theorem would imply that the 'electric field' generated by each cell would vanish outside the cell, i.e, there would be no interaction between the cells. The cells are, of course, never strictly disc shaped, but among the three possibilities (triangular, rectangular and hexagonal) for tilings of the plane with regular polygonal cells the hexagonal ones have the least mutual interaction energy. The vortices then sit on a triangular lattice. The interaction between the cells, although not zero, is small because the cells have only a quadrupole moment or higher and no dipole moment. The hexagonal cell is distinguished by having the smallest multipole moments ${ }^{8}$

The upper bound to the energy proved in [15] was achieved along the line just described. The lower bound relies on constructions and theorems from Ginzburg-Landau theory [59].

\subsubsection{Emergence of a 'giant vortex'}

As already mentioned, a transition to a new phase takes place when $\Omega_{\text {rot }}$ is of the order $1 /\left(\varepsilon|\log \varepsilon|^{2}\right)$. Here a variational ansatz of the form

$$
\psi(\mathbf{r})=f(\mathbf{r}) \exp \left(\mathrm{i} \hat{\Omega}_{\text {rot }} \theta\right)
$$

with a real valued function $f$ and

$$
\hat{\Omega}_{\mathrm{rot}}=\Omega_{\mathrm{rot}}-O\left(\varepsilon^{-1}\right)
$$

\footnotetext{
8 Although this is in accord with the apparent arrangement of vortices as observed in experiments, it is not a proof that the vortices must sit on a triangular lattice. First, it is not proved that the vortices sit on a regular lattice at all, and secondly, it turns out that the order of the energy considered in Theorem 5.3 does not distinguish between different regular lattices that all lead to cells with vanishing dipole moment. The energetic distinction between different regular arrangements is a delicate higher order effect. This topic has recently been analyzed in 60].
} 
gives a lower energy that the vortex lattice ansatz leading to 105, 106. This does not prove, however, that the energy $E^{\mathrm{gv}}$ of the ansatz (114) gives a good approximation to the energy of the true minimizer, nor that the latter is free of vortices in the bulk. That both statements are true is the content of the following theorems [14]:

Theorem 5.4 (Energy in the giant vortex regime) There is a constant $0<\Omega_{\mathrm{c}_{3}}<\infty$ such that for $\Omega=\Omega_{0}\left(\varepsilon|\log \varepsilon|^{2}\right)^{-1}$ with $\Omega_{0}>\Omega_{c_{3}}$ the ground state energy is

$$
E^{\mathrm{GP}}=E^{\mathrm{gv}}-O\left(|\log \varepsilon|^{3 / 2} / \varepsilon^{2}\right) .
$$

Theorem 5.5 (Absence of vortices in the bulk) There is an annulus $\mathcal{A}$ of width $O\left(\left(\varepsilon \Omega_{\mathrm{rot}}\right)^{-1}\right)$ with $\int_{\mathcal{A}}\left|\psi^{\mathrm{GP}}\right|^{2}=1-o(1)$ such that for $\Omega$ as above and $\varepsilon$ sufficiently small the minimizer $\psi^{\mathrm{GP}}$ is free of zeros in the annulus.

The proof, in particular of Theorem 5.5. is surprisingly difficult but a heuristic explanation for the transition at $\Omega_{\mathrm{rot}} \sim 1 /\left(\varepsilon^{2}|\log \varepsilon|\right)$ can be given by exploiting the electrostatic analogy:

Consider the variational ansatz (114) and interpret $\hat{\Omega}_{\text {rot }}$ as a 'charge' situated at the origin. The 'electric field' generated this charge exactly cancels, in the annulus $\mathcal{A}$, the 'electric field' generated in the annulus by the uniform charge density $\Omega_{\text {rot }} / \pi$ of the 'hole' (by Newton's theorem), due to the vector potential. However, the 'charge' corresponding to the vector potential in the annulus is not cancelled, and this 'residual charge' is

$$
\sim \Omega_{\text {rot }} \times\left(\varepsilon \Omega_{\text {rot }}\right)^{-1}=\varepsilon^{-1} .
$$

The electrostatic energy of this residual charge distribution is $\sim \varepsilon^{-2}$.

Creating a vortex in the annulus neutralizes one charge unit and thus reduces the electrostatic energy by $\varepsilon^{-1}$. On the other hand, the cost of a vortex is $\sim f^{2}|\log \varepsilon|$, and we have $f^{2} \sim\left(\varepsilon \Omega_{\text {rot }}\right)$, so the cost of a single vortex in the bulk is

$$
\sim \varepsilon \Omega_{\mathrm{rot}}|\log \varepsilon| \text {. }
$$

Gain and cost are comparable if $\varepsilon^{-1} \sim \varepsilon \Omega_{\text {rot }}|\log \varepsilon|$, i.e., for

$$
\Omega_{\mathrm{rot}} \sim \frac{1}{\varepsilon^{2}|\log \varepsilon|} .
$$

If $\Omega_{\text {rot }}$ is smaller it still pays to create vortices also in the annulus, but if $\Omega_{\text {rot }}$ is larger, the cost outweighs the gain and the annulus is vortex free.

This picture is substantiated by the rigorous analysis in [14] and it has been generalized to other anharmonic traps besides the 'flat' case in 9. Here some interesting new features concerning the size of the vortices and the shape of the density distribution in the annulus appear. See [10, 11] for surveys on this topic. 


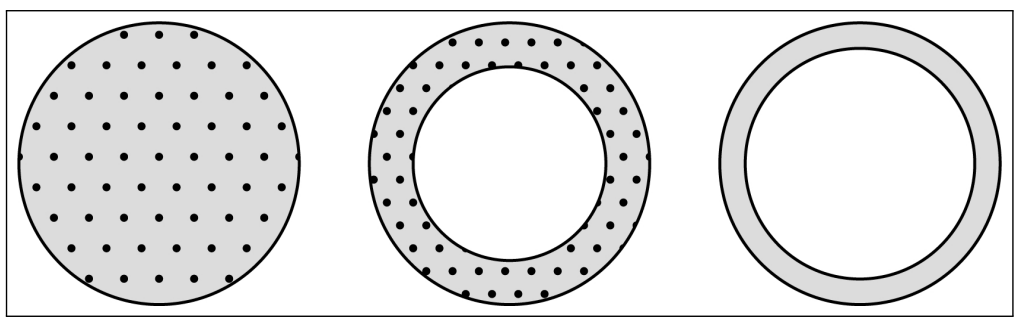

Fig. 3 The transition to a giant vortex.

\subsubsection{Summary on vortices}

The emergence of single vortices, vortex lattices and a 'giant vortex' state for a condensate in a rapidly rotating anharmonic trap can be understood by asymptotic analysis of the GP equation. When both the coupling constant $1 / \varepsilon^{2}$ and the rotational velocity $\Omega_{\text {rot }}$ are large the picture is as follows (in a 'flat' trap):

- Single vortices for $\Omega_{\text {rot }} \sim|\log \varepsilon|$

- A vortex lattice for $|\log \varepsilon| \ll \Omega_{\text {rot }} \ll 1 /\left(\varepsilon^{2}|\log \varepsilon|\right)$

- A 'hole' due to centrifugal forces for $1 / \varepsilon \lesssim \Omega_{\text {rot }}$.

- A 'giant vortex' for $1 /\left(\varepsilon^{2}|\log \varepsilon|\right) \lesssim \Omega_{\text {rot }}$

\section{Rapid Rotation and Confinement to the Lowest Landau Level}

One of the most striking phenomena in condensed matter physics is the Fractional Quantum Hall Effect (FQHE) for charged fermions in strong magnetic fields 67] that still, after decades of research, poses many challenging questions. It has been recognized for some time that bosonic analogues of the FQHE can be studied in cold quantum gases set in rapid rotation, see [68, 8 and references cited therein. In this section the focus will be on one aspect of the Quantum Hall Physics of cold bosons: The emergence of strongly correlated many-body states through appropriate tuning of the parameters.

The starting point is the many-body Hamiltonian 83 in the rotating frame that we recall for convenience:

$$
H_{N}=\sum_{j=1}^{N}\left(-\frac{1}{2} \nabla_{j}^{2}+V\left(\mathbf{x}_{j}\right)-\mathbf{L}_{j} \cdot \boldsymbol{\Omega}\right)+\sum_{1 \leq i<j \leq N} v\left(\left|\mathbf{x}_{i}-\mathbf{x}_{j}\right|\right) .
$$


In contrast to Section 5 we now consider the case when $V$ is a quadratic potential in the direction $\perp$ to the rotation axis,

$$
V(\mathbf{x})=\frac{1}{2} \Omega_{\text {trap }}^{2} r^{2}+V^{\|}\left(x_{3}\right),
$$

with $r^{2}=x_{1}^{2}+x_{2}^{2}$ and the angular velocity $\Omega_{\text {rot }}$ approaches the frequency of the potential $\Omega_{\text {trap }}$ from below. When writing the Hamiltonian with this potential in a 'magnetic' form, it is convenient to define the vector potential in a different way than in (85), namely

$$
\mathbf{A}(\mathbf{x})=\Omega_{\text {trap }}\left(x_{2},-x_{1}, 0\right)
$$

rather than $\mathbf{A}(\mathbf{x})=\Omega_{\text {rot }}\left(-x_{2}, x_{1}\right)$ as in $(85)$. With this definition the Hamiltonian takes the form

$$
H_{N}=\sum_{j=1}^{N}\left\{\frac{1}{2}\left(\mathrm{i} \nabla_{j}+\mathbf{A}\left(\mathbf{x}_{j}\right)\right)^{2}+\omega \mathbf{e}_{3} \cdot \mathbf{L}_{j}+V^{\|}\left(x_{3}\right)\right\}+\sum_{i<j} v\left(\left|\mathbf{x}_{i}-\mathbf{x}_{j}\right|\right)
$$

with

$$
\omega:=\Omega_{\text {trap }}-\Omega_{\text {rot }}>0 .
$$

\subsection{Confinement to the Lowest Landau Level, 1-Particle Case}

Consider now the one-particle Hamiltonian

$$
H_{1}=\frac{1}{2}\left(\mathrm{i} \nabla_{\perp}+\mathbf{A}(\mathbf{x})\right)^{2}+\omega \mathcal{L}-\frac{1}{2} \partial_{3}^{2}+V^{\|}\left(x_{3}\right)
$$

where we have written $\mathcal{L}=\mathbf{e}_{3} \cdot \mathbf{L}$ and $\nabla_{\perp}=\left(\partial_{1}, \partial_{2}\right)$. This is a sum of three commuting operators,

$$
\frac{1}{2}\left(\mathrm{i} \nabla_{\perp}+\mathbf{A}(\mathbf{x})\right)^{2}, \quad \omega \mathcal{L} \quad \text { and } \quad-\frac{1}{2} \partial_{3}^{2}+V^{\|}\left(x_{3}\right) .
$$

The spectrum of $\frac{1}{2}\left(\mathrm{i} \nabla_{\perp}+\mathbf{A}(\mathbf{x})\right)^{2}$ is

$$
\left(n+\frac{1}{2}\right) 2 \Omega_{\text {trap }}, \quad n=0,1,2, \ldots, \quad \text { called Landau levels. }
$$

The spectrum of $\omega \mathcal{L}$ is

$$
\ell \omega, \quad \ell=0, \pm 1, \pm 2 \ldots
$$

and $-\frac{1}{2} \partial_{3}^{2}+V^{\|}\left(x_{3}\right)=: h^{\|}$has a spectral gap, $e^{\|}>0$, above its ground state. 
For $\omega \ll \min \left\{\Omega_{\text {trap }}, e^{\|}\right\}$it is natural to restrict attention to states with $n=0$, and the motion in the $x_{3}$-direction is 'frozen' in the ground state of $h^{\|}$. From now on we choose units so that $\Omega_{\text {trap }}=1.9$

Replacing $\left(x_{1}, x_{2}\right)$ by the complex coordinate $z=x_{1}+\mathrm{i} x_{2}$ and denoting $\partial=\frac{1}{2}\left(\partial_{1}-\mathrm{i} \partial_{2}\right), \bar{\partial}=\frac{1}{2}\left(\partial_{1}+\mathrm{i} \partial_{2}\right)$ we can write

$$
\frac{1}{2}\left(\mathrm{i} \nabla_{\perp}+\mathbf{A}(\mathbf{x})\right)^{2}=2\left(\hat{a}^{\dagger} \hat{a}+\frac{1}{2}\right)
$$

with

$$
\hat{a}^{\dagger}:=\frac{1}{2}(-2 \partial+\bar{z}), \quad \hat{a}:=\frac{1}{2}(2 \bar{\partial}+z) .
$$

These operators satisfy the canonical commutation relations

$$
\left[\hat{a}, \hat{a}^{\dagger}\right]=1 .
$$

The operators

$$
\hat{b}^{\dagger}:=\frac{1}{2}(-2 \bar{\partial}+z), \quad \hat{b}:=\frac{1}{2}(2 \partial+\bar{z})
$$

also satisfy the canonical commutation relations and commute with $\hat{a}$ and $\hat{a}^{\dagger}$. They correspond to a replacement $\mathbf{A} \rightarrow-\mathbf{A}$ :

$$
\frac{1}{2}\left(\mathrm{i} \nabla_{\perp}-\mathbf{A}(\mathbf{x})\right)^{2}=2\left(\hat{b}^{\dagger} \hat{b}+\frac{1}{2}\right) .
$$

Moreover,

$$
\hat{b}^{\dagger} \hat{b}-\hat{a}^{\dagger} \hat{a}=z \partial-\bar{z} \bar{\partial}=\mathcal{L} .
$$

Hence the eigenvalues of $\hat{a}^{\dagger} \hat{a}$ are infinitely degenerate and the degenerate eigenstates can be labelled by eigenvalues of either $\hat{b}^{\dagger} \hat{b}$ or $\mathcal{L}$.

\subsubsection{Bargmann space}

The lowest eigenvalue of $\hat{a}^{\dagger} \hat{a}$ is zero. The corresponding eigenfunctions $\psi(z, \bar{z})$ are solutions of the equation $\hat{a} \psi=0$, i.e.,

$$
\bar{\partial} \psi(z, \bar{z})=-\frac{1}{2} z \psi(z, \bar{z}) .
$$

Thus

$$
\psi(z, \bar{z})=\varphi(z) \exp \left(-|z|^{2} / 2\right)
$$

with $\bar{\partial} \varphi(z)=0$, i.e., $\varphi$ is an analytic function of $z$.

In the lowest Landau level (LLL) we are thus led to consider the Bargmann space $\mathcal{B}$ [4, 24] of analytic functions $\varphi$ such that

\footnotetext{
${ }^{9}$ Since $|\nabla \times \mathbf{A}|=2 \Omega_{\text {trap }}$ this means that the 'magnetic field' unit is 2 rather than 1 . For this reason some formulas in the sequel may differ by powers of 2 from the ones customary in the theory of the FQHE for electrons.
} 


$$
\langle\varphi, \varphi\rangle:=\int|\varphi(z)|^{2} \exp \left(-|z|^{2}\right) \mathrm{d}^{2} z<\infty
$$

where $\mathrm{d}^{2} z$ denotes the Lebesgue measure on $\mathbb{C}$ (regarded as $\mathbb{R}^{2}$ ).

The Bargmann space is a Hilbert space with scalar product

$$
\langle\varphi, \psi\rangle:=\int \bar{\varphi}(z) \psi(z) \exp \left(-|z|^{2}\right) \mathrm{d}^{2} z
$$

On $\mathcal{B}$ the angular momentum operator is $\mathcal{L}=z \partial$. Moreover, for $\varphi \in \mathcal{B}$,

$$
\langle\varphi, \mathcal{L} \varphi\rangle=\int\left(|z|^{2}-1\right)|\varphi(z)|^{2} \exp \left(-|z|^{2}\right) \mathrm{d}^{2} z
$$

The eigenvalues of $\mathcal{L}$ restricted to $\mathcal{B}$ are $\ell=0,1,2, \ldots$ with corresponding normalized eigenfunctions

$$
\varphi_{\ell}(z)=(\pi \ell !)^{-1 / 2} z^{\ell}
$$

Note that $\left|\varphi_{\ell}(z)\right|^{2} e^{-|z|^{2}}$ has in the radial variable a maximum at $r_{\ell}=\sqrt{\ell}$. The density of states per unit area is therefore $1 / \pi$.

\subsection{Confinement to the Lowest Landau Level, N-particle Case}

We now come to the $N$-body problem for bosons in the LLL. The relevant Hilbert space is

$$
\mathcal{B}_{N}=\mathcal{B}^{\otimes_{\mathrm{symm}}^{N}}
$$

i.e., it consists of symmetric, analytic functions $\psi$ of $z_{1}, \ldots, z_{N}$ such that

$$
\int_{\mathbb{C}^{N}}\left|\psi\left(z_{1}, \ldots, z_{N}\right)\right|^{2} \exp \left(-\sum_{j=1}^{N}\left|z_{j}\right|^{2}\right) \mathrm{d}^{2} z_{1} \cdots \mathrm{d}^{2} z_{N}<\infty .
$$

As next we take the interaction into account, i.e., consider a suitable image of $\sum_{1 \leq i<j \leq N} v\left(\left|\mathbf{x}_{i}-\mathbf{x}_{j}\right|\right)$ as an operator on $\mathcal{B}_{N}$.

\subsubsection{Contact interaction}

For short range, nonnegative interaction potentials $v$ it was shown in 39] that for $\omega a \ll 1$, with $a$ the scattering length of $v$ the motion is indeed restricted to the 2D LLL, and moreover that $v\left(\mathbf{x}_{i}-\mathbf{x}_{j}\right)$ can be replaced by $g \delta\left(z_{i}-z_{j}\right)$ 
with 10

$$
g \sim a \sqrt{e^{\|}}>0
$$

Such a contact potential is perfectly acceptable for analytic functions and is even given by a bounded operator on the Bargmann space:

Define $\delta_{12}$ on $\mathcal{B}_{2}$ by

$$
\delta_{12} \varphi\left(z_{1}, z_{2}\right)=\frac{1}{2 \pi} \varphi\left(\frac{1}{2}\left(z_{1}+z_{2}\right), \frac{1}{2}\left(z_{1}+z_{2}\right)\right) .
$$

Then a simple computation, using the analyticity of $\varphi$, shows that

$$
\left\langle\varphi, \delta_{12} \varphi\right\rangle=\int_{\mathbb{C}}|\varphi(z, z)|^{2} \exp \left(-2|z|^{2}\right) \mathrm{d}^{2} z .
$$

Replacing $v$ by the contact interaction, the effective Hamiltonian on Bargmann space becomes (apart from an additive constant)

$$
H_{N}^{2 \mathrm{D}}=\omega \mathcal{L}_{N}+g \mathcal{I}_{N}
$$

with

$$
\mathcal{L}_{N}=\sum_{i=1}^{N} z_{i} \partial_{i} \quad \mathcal{I}_{N}=\sum_{i<j} \delta_{i j} .
$$

An important feature of the Hamiltonian 146 is that the operators $\mathcal{L}_{N}$ and $\mathcal{I}_{N}$ commute. The lower boundary of (the convex hull of) their joint spectrum in a plot with angular momentum as the horizontal axis is called the yrast curve. See Fig. 4 and 68 for its qualitative features.

As a function of the eigenvalues $L$ of $\mathcal{L}_{N}$ the yrast curve $I(L)$ is decreasing from $I(0)=(4 \pi)^{-1} N(N-1)$ to $I(N(N-1))=0$. The monotonicity follows from the observation that if a simultaneous eigenfunction of $\mathcal{L}_{N}$ and $\mathcal{I}_{N}$ is multiplied by the center of mass, $\left(z_{1}+\cdots+z_{N}\right) / N$, the interaction is unchanged while the angular momentum increases by one unit.

For a given ratio $\omega / g$ the ground state of (146) (in general not unique) is determined by the point(s) on the yrast curve where a supporting line has slope $-\omega / g$. The ground state energy is

$$
E(N, \omega, g)=\min _{L}(\omega L+g I(L)) .
$$

The filling factor of a state with angular momentum $L$ is defined as

$$
\nu=\frac{N(N-1)}{2 L}=\frac{N}{N_{\mathrm{v}}}
$$

${ }^{10}$ Note that this $g$ is, by definition, not proportional to $N$, in contrast to the previous coupling constant $g$ in 48 . 
where $N_{\mathrm{v}}=2 L /(N-1)$ is the number of vortices. The filling factor of the ground state depends on the ratio $\omega / g$ and varies from $\infty$ to 0 as the ratio decreases and the angular momentum increases.

\subsubsection{The GP regime}

A rough estimate for the radius $R$ of the system, assuming that kinetic and interaction energy are of the same order of magnitude, gives

$$
R \sim(N g / \omega)^{1 / 4} \quad \text { and } \quad L \sim N \Omega R^{2} \sim N(N g / \omega)^{1 / 2} .
$$

Thus, if If $\omega / g \gg N^{-1}$ then $L \ll N^{2}$ and $\nu \gg 1$. In this case the ground state can be shown to be well described by an uncorrelated Hartree state $\left(\varphi^{\mathrm{GP}}\right)^{\otimes N}$ where $N^{1 / 2} \varphi^{\mathrm{GP}}$ minimizes the GP energy functional

$$
\mathcal{E}^{\mathrm{GP}}[\varphi]=\omega\langle\varphi, \mathcal{L} \varphi\rangle+\frac{g}{2} \int_{\mathbb{C}}|\varphi(z)|^{4} \exp \left(-2|z|^{2}\right) \mathrm{d}^{2} z
$$

under the condition $\int|\varphi|^{2} \exp \left(-|z|^{2}\right) \mathrm{d}^{2} z=N$ with energy

$$
E^{\mathrm{GP}}(N, \omega, g)=N E^{\mathrm{GP}}(1, \omega, N g) .
$$

Note that only analytic functions in the Bargmann space $\mathcal{B}$ are allowed as trial functions for an upper bound to this energy.

More precisely, the following holds [47]:

Theorem 6.1 (GP limit theorem in LLL) For every $c>0$ there is a $C<\infty$ such that

$$
E^{\mathrm{GP}}(N, \omega, g) \geq E(N, \omega, g) \geq E^{\mathrm{GP}}(N, \omega, g)\left(1-C(g / N \omega)^{1 / 10}\right)
$$

provided $g N / \omega>c$.

The lower bound covers the whole regime $L \ll N^{2}$, i.e., $\nu \gg 1$, but the GP description might have a wider range of applicability. The proof uses similar techniques as in [42] for the 3D GP limit theorem at fixed $\Omega$ and $N a$, in particular coherent states.

\subsubsection{Gaps}

For every value of the angular momentum $L$ the interaction operator $\mathcal{I}_{N}$ has a nonzero spectral gap above 0

$$
\Delta_{N}(L)=\inf \left\{\operatorname{spec} \mathcal{I}_{N}\left\lceil_{\mathcal{L}_{N}=L} \backslash\{0\}\right\}>0 .\right.
$$


The gap, and hence the Yrast curve $I(L)$, are monotonously decreasing with $L$ for the reason already mentioned: The angular momentum of an eigenstate of $\mathcal{I}_{N}$ can be increased by one unit by multiplying the wave function with the center of mass coordinate $\left(z_{1}+\cdots+z_{N}\right) / N$. This does not change the interaction energy and leads to a family of 'daughter states' for each state on the yeast curve. There is numerical and some theoretical evidence that

$$
\Delta_{N}(L) \geq \Delta_{N}(N(N-1)-N)=\Delta:=\min _{L^{\prime}} \Delta_{N}\left(L^{\prime}\right)>0
$$

for all $L$ independently of $N$ but this is still not proved. We shall call the validity of 155 the gap conjecture.

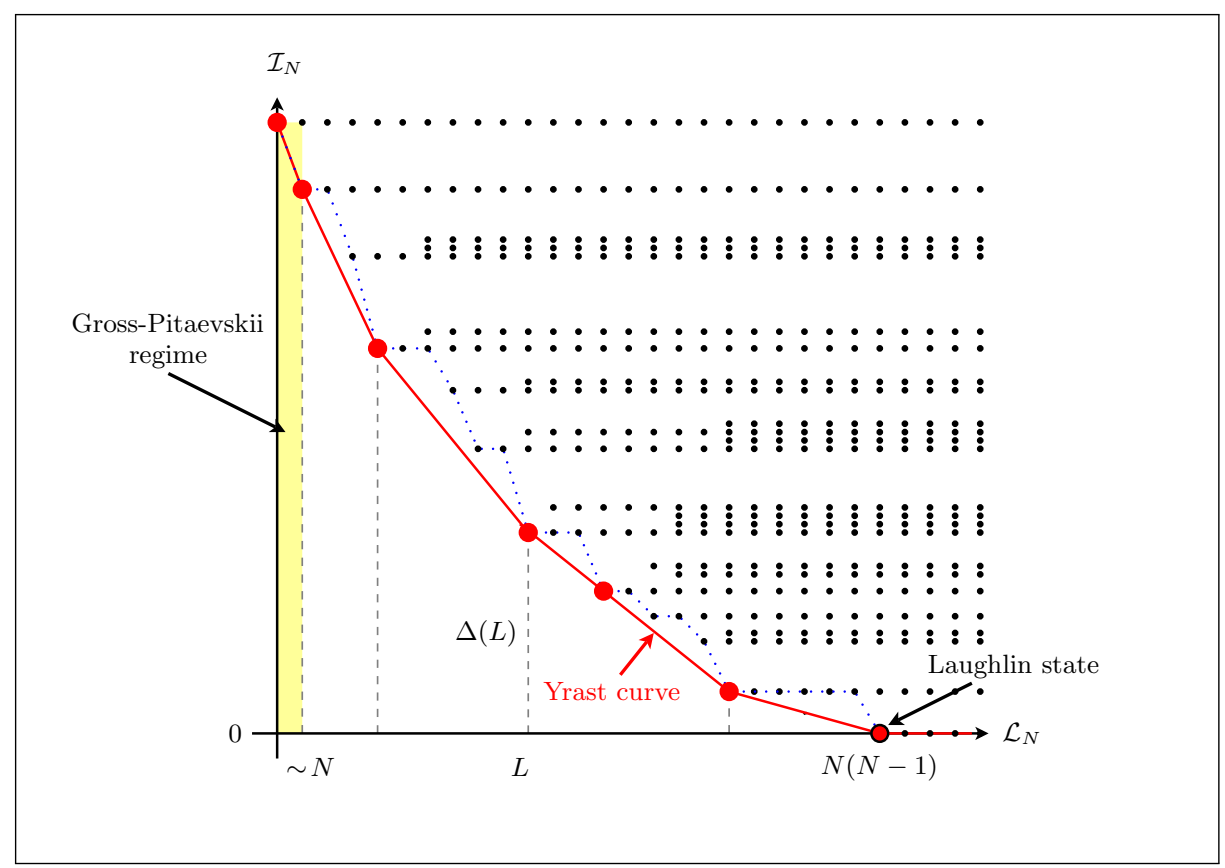

Fig. 4 The joint spectrum of $\mathcal{L}_{N}$ and $\mathcal{I}_{N}$ (adapted from [39]).

\subsection{Passage to the Laughlin state}

As the filling factor decreases the ground state becomes increasingly correlated. The exact ground states are largely unknown (except for $L \leq N$ [51]), but candidates of states with various rational filling factors (composite 
fermion states, Moore-Read states, Read-Rezayi states,...) for energy upper bounds have been suggested and studied, See e.g. the review article 8$]$.

If $\omega / g<\Delta / N^{2}$ one reaches the Laughlin state with filling factor $\frac{1}{2}$ whose wave function in Bargmann space is

$$
\psi_{\text {Laughlin }}\left(z_{1}, \ldots, z_{N}\right)=c \prod_{i<j}\left(z_{i}-z_{j}\right)^{2} .
$$

It has interaction energy 0 , angular momentum $L=N(N-1)$.

The limit $\omega \rightarrow 0$, keeping $\omega>0$, is experimentally very delicate, however. For stability, but also to study new effects, we consider now a modification of the Hamiltonian by adding a small anharmonic term:

$$
H_{N}^{2 \mathrm{D}} \rightarrow H_{N}^{2 \mathrm{D}}+k \sum_{i=1}^{N}\left|z_{i}\right|^{4}
$$

with a new parameter $k>0$. The potential $|z|^{4}$ can be expressed through $\mathcal{L}$ and $\mathcal{L}^{2}$ on Bargmann space, because with $\mathcal{L}=z \partial$ we have by partial integration, using the analyticity of $\varphi$,

$$
\langle\varphi, \mathcal{L} \varphi\rangle=\int|\varphi(z)|^{2}\left(|z|^{2}-1\right) \exp \left(-|z|^{2}\right) \mathrm{d}^{2} z
$$

and

$$
\left\langle\varphi, \mathcal{L}^{2} \varphi\right\rangle=\int\left(|z|^{4}-3|z|^{2}+1\right)|\varphi(z)|^{2} \exp \left(-|z|^{2}\right) \mathrm{d}^{2} z
$$

Thus the modified Hamiltonian, denoted again by $H_{N}^{2 \mathrm{D}}$, can be written (up to an additive constant)

$$
H_{N}^{2 \mathrm{D}}=(\omega+3 k) \mathcal{L}_{N}+k \sum_{i=1}^{N} \mathcal{L}_{(i)}^{2}+g \mathcal{I}_{N} .
$$

\subsubsection{Fully correlated states}

The Bargmann space $\mathcal{B}^{N}$ with the scalar product 142 is naturally isomorphic to the Hilbert space $\left.\mathcal{H}_{\mathrm{LLL}}^{N} \subset L^{2}(\mathbb{C})^{\otimes_{s} N}, \mathrm{~d}^{2 N} z\right)$ consisting of wave functions of the form

$$
\Psi\left(z_{1}, \ldots, z_{N}\right)=\psi\left(z_{1}, \ldots, z_{N}\right) \exp \left(-\sum_{j}\left|z_{j}\right|^{2} / 2\right), \quad \psi \in \mathcal{B}_{N}
$$

with the standard $L^{2}$ scalar product. The energy can accordingly be considered as a functional on this space, 


$$
\mathcal{E}[\Psi]=\int V_{\omega, k}(z) \rho_{\Psi}(z)+\left\langle\Psi, \mathcal{I}_{N} \Psi\right\rangle
$$

where $\rho_{\Psi}$ is the one-particle density of $\Psi$ with the normalization $\int \rho_{\Psi}(z) \mathrm{d}^{2} z=$ $N$ and the potential is

$$
V_{\omega, k}(z)=\omega|z|^{2}+k|z|^{4}
$$

Note that now $\omega<0$ is allowed, provided $k>0$.

We shall call states with vanishing interaction energy, i.e., $\Psi \in \operatorname{ker} \mathcal{I}_{N}$ fully correlated, because the particles stay away from each other in the sense that the wave function vanishes if $z_{i}=z_{j}$ for some pair $i \neq j$, in sharp contrast to a fully uncorrelated Hartree state. The fully correlated states in $\mathcal{H}_{\mathrm{LLL}}^{N}$ are of the form

$$
\Psi\left(z_{1}, \ldots z_{N}\right)=\phi\left(z_{1}, \ldots, z_{N}\right) \Psi_{\text {Laugh }}\left(z_{1}, \ldots z_{N}\right)
$$

with $\phi$ symmetric and analytic, and the Laughlin state

$$
\Psi_{\text {Laugh }}\left(z_{1}, \ldots z_{N}\right)=c \prod_{i<j}\left(z_{i}-z_{j}\right)^{2} e^{-\sum_{j=1}^{N}\left|z_{j}\right|^{2} / 2} .
$$

For an intuitive picture of the Laughlin state the following analogy may be helpful. The density $\left|\Psi_{\text {Laugh }}\left(z_{1}, \ldots z_{N}\right)\right|^{2}$ assigns probabilities to the possible configurations of $N$ points moving in the plane. The points like to keep a distance at least of order 1 from each other because the factors $\left|z_{i}-z_{j}\right|^{4}$ strongly reduce the probability when the particles are close. On the other hand the damping due to the gaussian favors a tight packing of the 'balls' of size $O(1)$ around the individual particles. The motion is strongly correlated in the sense that if one ball moves, all the other have also to move in order to satisfy these constraints ${ }^{11}$

For the Hamiltonian without the anharmonic addition to the potential the Laughlin state is an exact fully correlated ground state with energy 0 and angular momentum $L_{\text {Laugh }}=N(N-1)$. This is not true for $k \neq 0$ because $\sum_{i=1}^{N} \mathcal{L}_{(i)}^{2}$ does not commute with $\mathcal{I}_{N}$. Note, however, that $\mathcal{L}_{N}$ still commutes with the Hamiltonian.

We now address the following question: Under what conditions is it possible to tune the parameters so that the ground state $\Psi_{0}$ of $(160)$ becomes fully correlated for $N \rightarrow \infty$ ? The following theorem, proved in [57, gives sufficient conditions for this to happen. In order to state it as simply as possible we shall assume the 'gap conjecture' of Subsection 6.2.3. This conjecture is not really needed, however, because is possible to replace the assumed universal gap $\Delta$ by other gaps depending on the parameters, cf. Eq. (IV.5) in [57.

11 A colony of Emperor Penguins, sticking tightly together to survive the antarctic winter, but where the individual penguins are constantly on the move exchanging places with each other, is not a bad picture to have in mind! 


\section{Theorem 6.2 (Criteria for full correlation)}

$$
\left\|P_{\left(\operatorname{Ker} \mathcal{I}_{N}\right)^{\perp}} \Psi_{0}\right\| \rightarrow 0
$$

in the limit $N \rightarrow \infty, \omega, k \rightarrow 0$ if one of the following conditions hold:

- $\omega \geq 0$ and $\omega N^{2}+k N^{3} \ll g \Delta$.

- $0 \geq \omega \geq-2 k N$ and $N\left(\omega^{2} / k\right)+\omega N^{2}+k N^{3} \ll g \Delta$.

- $\omega \leq-2 k N,|\omega| / k \lesssim N^{2}$ and $k N^{3} \ll g \Delta$

- $\omega \leq-2 k N,|\omega| / k \gg N^{2}$ and $|\omega| N \ll g \Delta$

Note: For $k=0$ the first item is just the sufficient condition for the passage to the Laughlin state, $\omega / g<\Delta / N^{2}$, while the other conditions are void because $\omega<0$ is only allowed if $k>0$.

The proof of the Theorem is based on the following two items

- A lower bound for the ground state energy at fixed angular momentum $L$ :

$$
E_{0}(L) \geq(\omega+3 k) L+k \frac{L^{2}}{N} .
$$

- An upper bound for the energy of suitable trial functions.

The first bound is quite simple; it follows essentially from

$$
\sum_{i} \mathcal{L}_{(i)}^{2} \geq \frac{1}{N}\left(\sum_{i} \mathcal{L}_{(i)}\right)^{2}
$$

that holds because $\mathcal{L}_{(i)}$ and $\mathcal{L}_{(j)}$ commute for any $i, j$.

The upper bound is achieved by means of trial states of the form 'giant vortex times Laughlin', namely, with $m \geq 0$ and $c_{m, N}$ a normalization constant,

$$
\Psi_{\mathrm{gv}}^{(m)}\left(z_{1}, \ldots, z_{N}\right)=c_{m, N} \prod_{j=1}^{N} z_{j}^{m} \prod_{i<j}\left(z_{i}-z_{j}\right)^{2} e^{-\sum_{j=1}^{N}\left|z_{j}\right|^{2} / 2}
$$

For small $m$ these are Laughlin's 'quasi hole' states 36 but for $m \gtrsim N$, i.e., $m N \gtrsim N^{2}=$ angular momentum of the Laughlin state, the label 'giant vortex' appears more appropriate. Note, however, that mathematically and physically these states are rather different from the previously considered uncorrelated giant vortex states in Section 5.6.2.

The energy of the trial states can be estimated using properties of the angular momentum operators and the radial symmetry in each variable of 
$\prod_{j=1}^{N}\left|z_{j}\right|^{2 m} \times$ the gaussian measure. Optimizing the estimate over $m$ leads to

$$
m_{\mathrm{opt}}= \begin{cases}0 & \text { if } \omega \geq-2 k N \\ \frac{|\omega|}{2 k}-N & \text { if } \omega<-2 k N\end{cases}
$$

This is consistent with the picture that the Laughlin state is an approximate ground state in the first two cases of Theorem 1, in particular for negative $\omega$ as long as $|\omega| / k \lesssim N$. The angular momentum remains $O\left(N^{2}\right)$ in these cases.

When $\omega<0$ and $|\omega| /(k N)$ becomes large the angular momentum is approximately $L_{\mathrm{qh}}=O(N|\omega| / k) \gg N^{2}$, much larger than for the Laughlin state. A further transition at $|\omega| / k \sim N^{2}$ is manifest through the change of the subleading contribution to the energy of the trial functions. Its order of magnitude changes from $O\left(k N^{3}\right)$ to $O(|\omega| N)$ at the transition.

To obtain further insights into the physics of the transition we consider the density of the trial wave functions. This analysis [58] is based on the analogy of the $N$-particle density with the Gibbs distribution of a 2D Coulomb gas [36. Taking a mean field limit of this system brings out the essential features of the single particle density for large $N$, in particular its incompressibility.

\subsection{The N-particle Density as a Gibbs Measure}

We denote $\left(z_{1}, \ldots, z_{N}\right)$ by $Z$ for short and consider the scaled $N$ particle density (normalized to 1 )

$$
\rho_{N, m}(Z):=N^{N}\left|\Psi_{\mathrm{gv}}^{(m)}(\sqrt{N} Z)\right|^{2}
$$

We can write

$$
\begin{aligned}
\rho_{N, m}(Z) & =\mathcal{Z}_{N, m}^{-1} \exp \left(\sum_{j=1}^{N}\left(-N\left|z_{j}\right|^{2}+2 m \log \left|z_{j}\right|\right)+4 \sum_{i<j} \log \left|z_{i}-z_{j}\right|\right) \\
& =\mathcal{Z}_{N, m}^{-1} \exp \left(-\frac{1}{T} \mathcal{H}_{N, m}(Z)\right)
\end{aligned}
$$

with $T=N^{-1}$ and

$$
\mathcal{H}_{N, m}(Z)=\sum_{j=1}^{N}\left(\left|z_{j}\right|^{2}-\frac{2 m}{N} \log \left|z_{j}\right|\right)-\frac{4}{N} \sum_{i<j} \log \left|z_{i}-z_{j}\right| .
$$




\subsubsection{Plasma analogy and mean field limit}

The Hamilton function $\mathcal{H}_{N, m}(Z)$ defines a classical 2D Coulomb gas ('plasma', (jellium') in a uniform background of opposite charge and with a point charge $(2 m / N)$ at the origin, corresponding respectively to the $\left|z_{i}\right|^{2}$ and the $-\frac{2 m}{N} \log \left|z_{j}\right|$ terms.

The probability measure $\rho_{N, m}(Z)$ minimizes the free energy functional

$$
\mathcal{F}(\rho)=\int_{\mathbb{R}^{2 N}} \mathcal{H}_{N, m}(Z) \rho(Z)+T \int_{\mathbb{R}^{2 N}} \rho(Z) \log \rho(Z)
$$

for this Hamiltonian at $T=N^{-1}$.

The $N \rightarrow \infty$ limit is in this interpretation a mean field limit where at the same time $T \rightarrow 0$. It is thus not unreasonable to expect that for large $N$, and in an appropriate sense,

$$
\rho_{N, m} \approx \rho^{\otimes N}
$$

with a one-particle density $\rho$ minimizing a mean field free energy functional.

The mean field free energy functional is defined as

$$
\mathcal{E}_{N, m}^{\mathrm{MF}}[\rho]:=\int_{\mathbb{R}^{2}} W_{m} \rho-2 \int_{\mathbb{R}^{2}} \int_{\mathbb{R}^{2}} \rho(z) \log \left|z-z^{\prime}\right| \rho\left(z^{\prime}\right)+N^{-1} \int_{\mathbb{R}^{2}} \rho \log \rho
$$

with

$$
W_{m}(z)=|z|^{2}-2 \frac{m}{N} \log |z| .
$$

It has a minimizer $\rho_{N, m}^{\mathrm{MF}}$ among probability measures on $\mathbb{R}^{2}$ and this minimizer is in 58 proved to be a good approximation for the scaled 1-particle density of the trial wave function, i.e.,

$$
\rho_{N, m}^{(1)}(z):=\int_{\mathbb{R}^{2(N-1)}} \rho_{N, m}\left(z, z_{2}, \ldots, z_{N}\right) \mathrm{d}^{2} z_{2} \ldots \mathrm{d}^{2} z_{N} .
$$

(Recall the scaling: This density in the scaled variables $z$ is normalizes so that its integral is 1 . The corresponding density in the physical, unscaled variables $\zeta=\sqrt{N} z$ has total mass $N$.)

\subsubsection{Asymptotic formulas for the mean field density}

The picture of the 1-particle density that arises from asymptotic formulas for the mean-field density is as follows:

If $m \leq N^{2}$, then $\rho_{m}^{\mathrm{MF}}$ is well approximated by a density $\hat{\rho}_{m}^{\mathrm{MF}}$ that minimizes the mean field functional without the entropy term. 
It takes a constant valu $£^{12}(2 \pi)^{-1}$ (for all $N$ )on an annulus with inner and outer radii (in the scaled variables!)

$$
R_{-}=(m / N)^{1 / 2}, \quad R_{+}=(2+m / N)^{1 / 2}
$$

and is zero otherwise. The constant value is a manifestation of the incompressibility of the density of the trial state.

For $m \gtrsim N^{2}$ the entropy term dominates the interaction term $\iint \rho(z) \log \left|z-z^{\prime}\right| \rho\left(z^{\prime}\right)$. The density is well approximated by the Gaussian $\rho^{\text {th }}(z) \sim|z|^{2 m} \exp \left(-N|z|^{2}\right)$ that is centered around $(m / N)^{-1 / 2}$ but has maximal value $\sim N / m^{1 / 2} \ll 1$ for $m \gg N^{2}$.

As the parameters $\omega$ and $k$ tend to zero and $N$ is large the qualitative properties of the optimal trial wave functions thus exhibit different phases:

- The state changes from a pure Laughlin state to a modified Laughlin state with a 'hole' in the density around the center when $\omega$ is negative and $|\omega|$ exceeds $2 k N$.

- A further transition is indicated at $|\omega| \sim k N^{2}$. The density profile changes from being 'flat' to a Gaussian.

An intuitive understanding of these transitions may be obtained by employing the previous picture of the points $z_{i}$ as being the centers of essentially non overlapping balls of size $O(1)$. From the point of view of the plasma analogy the particles stay away from each other because of the repulsive Coulomb potential between them, while the attractive external potential due to the uniformly charged background keeps them as close together as possible. Modifying the wave function by a factor $\prod_{j} z_{j}^{m}$ has the effect of a repulsive charge of magnitude $m$ at the origin that pushes the particles (collectively!) away from the origin, creating a 'hole'. The effect of such a hole on the energy of the wave function in the trap potential is to increase the energy if $\omega$ is positive. Hence the ground state will not have a hole. If $\omega<0$ the effective trapping potential has a Mexican hat shape with a minimum away from the origin, but since no ball can move without 'pushing' all the other balls, it is too costly for the system to take advantage of this as long as $k$ stays above the critical value $|\omega| / 2 N$. For smaller $k$ a hole is formed. The balls remain densely packed until the minimum of the Mexican hat potential moves so far from the origin that an annulus of width $O(1)$ at the radius of the minimum can accommodate all $N$ balls. This happens for $k \lesssim|\omega| / N^{2}$. For smaller $k$ (larger radius) the balls need not be tightly packed in the annulus and the average local density decreases accordingly.

\footnotetext{
12 Note that this is a statement about the mean field density that is a good approximation in a weak sense (but not pointwise) to the true 1-particle density for large $N$. See 7] for numerical calculations of the true density for $N=400$.
} 

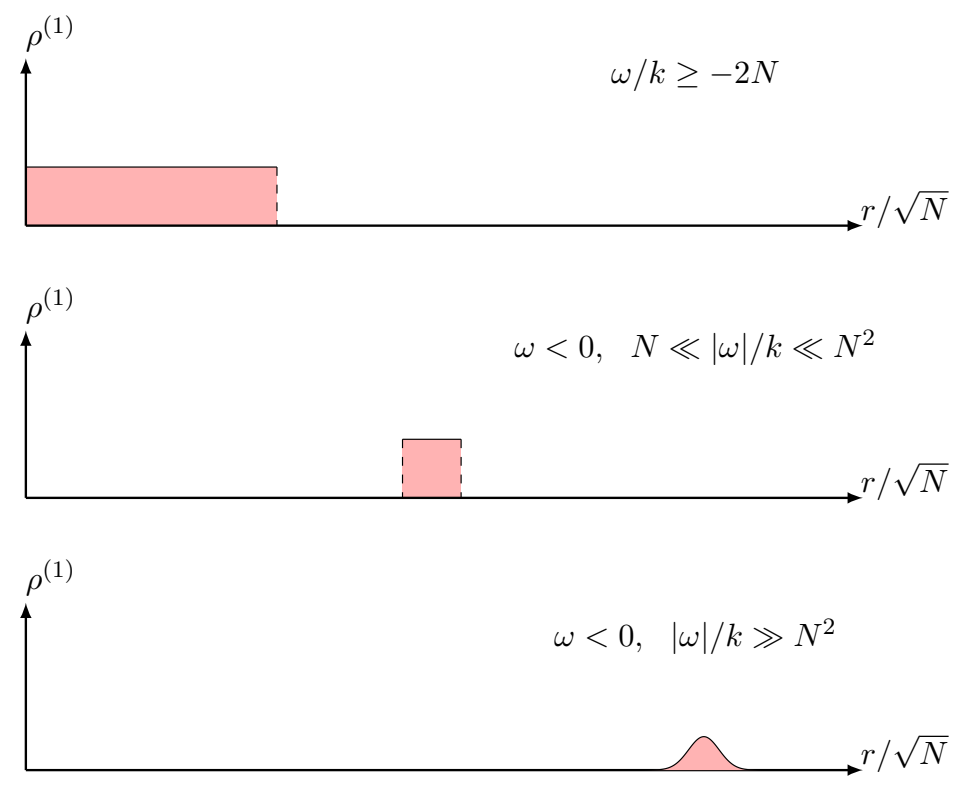

Fig. 5 The three phases of the density $\rho_{N, m}^{(1)}$ (not to scale).

\subsection{Summary and Conclusions}

The main conclusion from the analysis presented above of of many-body ground states in the lowest Landau level generated by fast rotation can be summarized as rolls:

- The parameter regime $g \ll N \omega$, i.e., $L \ll N^{2}$, can be described by a GP theory in the LLL.

- To enter the 'fully correlated' regime with $L \geq N(N-1)$ we have studied a rotating Bose gas in a quadratic plus quartic trap (coupling $k$ ) where the rotational frequency can exceed the frequency of the quadratic part of the trap, i.e, the frequency difference $\omega$ can be negative.

- Through the analysis of trial states for energy upper bounds and simple lower bounds we have obtained criteria for the ground state to be fully correlated in an asymptotic limit. The lower bounds, although not sharp, are of the same order of magnitude as the upper bounds. 
- The density of the wave functions can be analyzed through the plasma analogy. The character of the density changes at $|\omega| / k=O(N)$ and again at $|\omega| / k=O\left(N^{2}\right)$.

Acknowledgements I thank Nicolas Rougerie for valuable comments, Christian Köhler, Matthias Plaschke, Mathieu Lewin and Robert Seiringer for help with the figures, and the Austrian Science Fund (FWF) for support under Project P 22929N16.

\section{References}

1. A. Aftalion, Vortices in Bose-Einstein Condensates, Progress in Nonlinear Differential Equations and their Applications 67, Birkhäuser, Basel, 2006.

2. A. Aftalion, Q. Du Q, Vortices in a rotating Bose-Einstein condensate: Critical angular velocities and energy diagrams in the Thomas-Fermi regime, Phys. Rev. A 64, 063603 (2001).

3. A. Aftalion, R. L. Jerrard, J. Royo-Letelier, Non Existence of Vortices in the Small Density Region of a Condensate, J. Funct. Anal. 260, 2387-2406 (2011).

4. V. Bargmann, On a Hilbert Space of Analytic Functions and an Associated Integral Transform, Comm. Pure Appl. Math. 14, 187-214 (1961).

5. N.N. Bogoliubov, On the theory of superfluidity, Izv. Akad. Nauk USSR, 11, 77 (1947). Eng. Trans. J. Phys. (USSR), 11, 23 (1947). See also Lectures on quantum statistics, vol. 1, Gordon and Breach (1967).

6. J.-B. Bru, M. Correggi, P. Pickl, J. Yngvason, The TF Limit for Rapidly Rotating Bose Gases in Anharmonic Traps, Comm. Math. Phys. 280, 517-544 (2008).

7. O. Ciftuy, Monte Carlo study of Bose Laughlin wave function for filling factors 1/2, 1/4 and 1/6, Europhys. Lett. 74, 486-492 (2006).

8. N.R. Cooper, Rapidly Rotating Atomic Gases, Adv. Phys. 57, 539-616 (2008).

9. M. Correggi, F. Pinsker, N. Rougerie, J. Yngvason, Rotating superfluids in anharmonic traps: From vortex lattices to giant vortices, Phys. Rev. A 84, 053614 (2011).

10. M. Correggi, F. Pinsker, N. Rougerie, J. Yngvason, Vortex Phases of Rotating Superfluids, J. Phys. Conference Series 414, 012034 (2013).

11. M. Correggi, F. Pinsker, N. Rougerie, J. Yngvason, Giant vortex phase transition in rapidly rotating trapped Bose-Einstein condensates, Eur. Phys. J. Special Topics 217, 183-188 (2013).

12. M. Correggi, T. Rindler-Daller, J. Yngvason, Rapidly Rotating BoseEinstein Condensates in Homogeneous Traps, J. Math. Phys. 48, 102103 (2007).

13. M. Correggi, N. Rougerie, Inhomogeneous Vortex Patterns in Rotating Bose-Einstein Condensates, Commun. Math. Phys. 321, 817-860 (2013).

14. M. Correggi, N. Rougerie, J. Yngvason, The Transition to a Giant Vortex Phase in a Fast Rotating Bose-Einstein Condensate, Commun. Math. Phys. 303, 451-508 (2011).

15. M. Correggi, J. Yngvason, Energy and Vorticity in Fast Rotating BoseEinstein Condensates, J. Phys. A: Math. Theor. 41, 445002 (2008).

16. F.J. Dyson, Ground-State Energy of a Hard-Sphere Gas, Phys. Rev. 106, 20-26 (1957). 
17. A. Einstein, Quantentheorie des einatomigen idealen Gases, Sitzber. Kgl. Preuss. Akad. Wiss., 261-267 (1924), and 3-14 (1925).

18. L. Erdős, B. Schlein, H.T. Yau, Derivation of the Cubic Non-Linear Schrödinger Equation from Quantum Dynamics of Bose-Einstein Condensate, Invent. Math. 167, 515-614 (2007).

19. L. Erdős, B. Schlein, H.T. Yau, Ground state energy of a low-density Bose gas: A second order upper bound, Phys. Rev. A 78, 053627 (2008).

20. A.L. Fetter, Rotating Trapped Bose-Einstein Condensates, Rev. Mod. Phys. 81, 647-691 (2009).

21. A.L. Fetter, N. Jackson, S. Stringari, Rapid Rotation of a Bose-Einstein Condensate in a Harmonic Plus Quartic Trap, Phys. Rev. A 71, 013605 (2005).

22. U.R. Fischer, G. Baym, Vortex States of Rapidly Rotating Dilute BoseEinstein Condensates, Phys. Rev. Lett. 90, 140402 (2003).

23. H. Fu, E. Zaremba, Transition to the Giant Vortex State in a Harmonicplus-quartic Trap, Phys. Rev. A 73, 013614 (2006).

24. S. GiRvin, T. JACH, Formalism for the quantum Hall effect: Hilbert space of analytic functions, Phys. Rev. B 29, 5617-5625 (1984).

25. A. Giuliani, J. Seiringer, The Ground State Energy of the Weakly Interacting Bose Gas at High Density, J. Stat. Phys. 135, 915-934 (2009).

26. E.P. Gross, Structure of a Quantized Vortex in Boson Systems, Nuovo Cimento 20, 454-466 (1961).

27. E.P. Gross, Hydrodynamics of a superfluid condensate, J. Math. Phys. 4, 195-207 (1963).

28. K. Huang, Statistical Mechanics, Wiley, 1987.

29. K. Huang, C.N. Yang, Phys. Rev. 105, 767-775 (1957); T.D. Lee, K. Huang, C.N. Yang, Phys. Rev. 106, 1135-1145 (1957); K.A. Brueckner, K. SAWAdA, Phys. Rev. 106, 1117-1127, 1128-1135 (1957); S.T. Beliaev, Sov. Phys.-JETP 7, 299-307 (1958); T.T. Wu, Phys. Rev. 115, 1390 (1959); N. Hugenholtz, D. Pines, Phys. Rev. 116, 489 (1959); M. Girardeau, R. Arnowitt, Phys. Rev. 113, 755 (1959); T.D. LeE, C.N. Yang, Phys. Rev. 117, 12 (1960).

30. R. Ignat, V. Millot, The Critical Velocity for Vortex Existence in a Twodimensional Rotating Bose-Einstein Condensate, J. Funct. Anal. 233, 260306 (2006).

31. R. Ignat, V. Millot, Energy Expansion and Vortex Location for a Two Dimensional Rotating Bose-Einstein Condensate, Rev. Math. Phys. 18, 119162 (2006).

32. K. Kasamatsu, M. Tsubota, M. Ueda, Giant Hole and Circular Superflow in a Fast Rotating Bose-Einstein Condensate, Phys. Rev. A 66, 050606 (2002).

33. G.M. Kavoulakis, G. Baym, Rapidly Rotating Bose-Einstein Condensates in Anharmonic Potentials, New J. Phys. 5, 51.1-51.11 (2003).

34. W. Ketterle, N.J. van Druten, Bose-Einstein condensation of particles trapped in one or three dimensions, Pbys. Rev A. 54, 656-660 (1996).

35. W. Ketterle, N.J. van Druten, Evaporative Cooling of Trapped Atoms, in: B. Bederson, H. Walther, eds., Advances in Atomic, Molecular and Optical Physics, 37, 181-236, Academic Press (1996).

36. R. B. Laughlin, Anomalous quantum Hall effect: An incompressible quantum fluid with fractionally charged excitations, Phys. Rev. Lett. 50, 1395-1398 (1983).

37. J. O. LeE, J. Yin, A Lower Bound on the Ground State Energy of Dilute Bose Gas, J. Math. Phys. 51, 053302 (2010).

38. W. LENZ, Die Wellenfunktion und Geschwindigkeitsverteilung des entarteten Gases, Z. Phys. 56, 778-789 (1929). 
39. M. Lewin, R. Seiringer, Strongly Correlated Phases in Rapidly Rotating Bose Gases, J. Stat. Phys. 137 , 1040-1062 (2009).

40. E.H. Lieb, Simplified Approach to the Ground State Energy of an Imperfect Bose Gas, Phys. Rev. 130, 2518-2528 (1963). See also Phys. Rev. 133 (1964), A899-A906 (with A.Y. Sakakura) and Phys. Rev. 134 (1964), A312-A315 (with W. Liniger).

41. E.H. Lieb, Thomas-Fermi and related theories of atoms and molecules, Rev. Mod. Phys. 53, 603 (1981). Errata 54, 311 (1982).

42. E.H. Lieb, R. Seiringer, Proof of Bose-Einstein Condensation for Dilute Trapped Gases, Phys. Rev. Lett. 88, 170409-1-4 (2002).

43. E.H. Lieb, R. Seiringer, Derivation of the Gross-Pitaevskii Equation for Rotating Bose Gases, Comm. Math. Phys. 264, 505-537 (2006).

44. E.H. Lieb, R. Seiringer, J.P. Solovej, J. Yngvason, The Mathemat$i c s$ of the Bose Gas and its Condensation, Oberwolfach Seminar Series 34, Birkhäuser, Basel (2005). arXiv:cond-mat/0610117

45. E.H. Lieb, R. Seiringer, J. Yngvason, Bosons in a Trap: A Rigorous Derivation of the Gross-Pitaevskii Energy Functional, Phys. Rev. A. 61, 043602 (2000).

46. E.H. Lieb, R. Seiringer, and J. Yngvason, Poincaré Inequalities in Punctured Domains, Ann. Math. 158, 1067-1080 (2003).

47. E.H. Lieb, R. Seiringer, J. Yngvason, The Yrast Line of a Rapidly Rotating Bose Gas: The Gross-Pitaevskii Regime, Phys. Rev. A 79, 063626 (2009).

48. E.H. Lieb, J. Yngvason, Ground State Energy of the low density Bose Gas, Phys. Rev. Lett. 80, 2504-2507 (1998).

49. E.H. Lieb, J. Yngvason, The Ground State Energy of a Dilute Twodimensional Bose Gas, J. Stat. Phys. 103, 509 (2001).

50. L. Onsager, Remark in the discussion following a paper by C. J. Gorter on the two-fluid model of liquid helium, Supp. to Nuovo Cimento, 6, 249250 (1949).

51. T. Papenbrock, G.F. Bertsch, Rotational spectra of weakly interacting Bose-Einstein condensates, Phys. Rev. A 63, 023616 (2001).

52. P. PICKL, A simple derivation of mean field limits for quantum systems, Lett. Math. Phys. 97, 151-164 (2011).

53. C. Pethick, H. Sмith, Bose-Einstein Condensation of Dilute Gases, 2nd edition, Cambridge University Press (2008).

54. L.P. Pitaevskit, Vortex lines in an imperfect Bose gas, Sov. Phys. JETP. 13, 451-454 (1961).

55. L. Pitaevskit and S. Stringari, Bose-Einstein Condensation, Oxford Science Publications, Oxford (2003).

56. Rindler-Daller T., Vortices in rotating Bose-Einstein condensates confined in homogeneous traps, Physica A 387 1851-1874 (2008).

57. N. Rougerie, S. Serfaty, J. Yngvason, Quantum Hall states of bosons in rotating anharmonic traps, Phys. Rev. A 87, 023618 (2013)

58. N. Rougerie, S. Serfaty, J. Yngvason, Quantum Hall phases and plasma analogy in rotating trapped Bose gases, J. Stat. Phys DOI 10.1007/s 10955013-0766-0 (2013), arXiv: 1301.1043

59. E. SAndier, S. Serfaty, Vortices in the Magnetic GinzburgLandau Model, Progress in Nonlinear Differential Equations and their Applications 70, Birkhäuser, Basel, 2007, erratum available at http://www.ann.jussieu.fr/serfaty/publis.html

60. E. SANDIER, S. Serfaty, 2D Coulomb gases and the renormalized energy, arxiv 1201:3503.

61. M. Schick, Two-Dimensional System of Hard Core Bosons, Phys. Rev. A 3, 1067-1073 (1971). 
62. R. Seiringer, Gross-Pitaevskii Theory of the Rotating Bose Gas, Commun. Math. Phys. 229, 491-509 (2002).

63. R. Seiringer, Ground state asymptotics of a dilute, rotating gas, J. Phys. A: Math. Gen. 36, 9755-9778 (2003).

64. R. Seiringer, Dilute, Trapped Bose Gases and Bose-Einstein Condensation, in: Large Coulomb Systems, Lecture Notes Phys. 695, 251-276, J. Derezinski, H. Siedentop, eds., Springer (2006).

65. R. Seiringer, Cold Quantum Gases and Bose-Einstein Condensation, in: Quantum Many Body Systems, Lecture Notes in Mathematics 2051, 55-92, A. Guiliani, V. Mastropietro, J. Yngvason, eds., Springer (2012).

66. L. Spruch, L. Rosenberg, Upper bounds on scattering lengths for static potentials, Phys. Rev. 116, 1034 (1959).

67. H.L. Stormer, D.C. Tsui, A.C. Gossard, The fractional quantum Hall effect, Rev. Mod. Phys. 71, S298-S305 (1999).

68. S. Viefers, Quantum Hall physics in rotating Bose-Einstein condensates, $J$. Phys. C 12, 123202 (2008).

69. H.-T. YAU, J. Yin, Upper Bound for the Ground Energy of a Bose Gas, J. Stat. Phys. 136, 453-503 (2009).

70. V.I. Yukalov, Principal problems in Bose-Einstein condensation of dilute gases, Laser Phys. Lett. 1, 435-461 (2004). 